\title{
Infinitely many radial solutions for a sub-super critical $p$-Laplacian problem
}

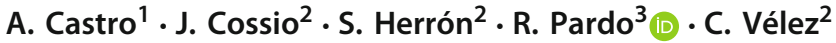

Received: 26 June 2018 / Accepted: 20 August 2019 / Published online: 30 August 2019

(c) Fondazione Annali di Matematica Pura ed Applicata and Springer-Verlag GmbH Germany, part of Springer Nature 2019

\begin{abstract}
We prove the existence of infinitely many sign-changing radial solutions for a $p$-sub-super critical $p$-Laplacian Dirichlet problem in a ball. We consider an equation defined by the $p$-Laplacian operator perturbed by a nonlinearity $g(u)$ that is $p$-subcritical at $+\infty$ and $p$ supercritical at $-\infty$. Our results extend those in Castro et al. (Electron. J. Differ. Equ. 2007(111):1-10, 2007) for the corresponding semilinear case and those of El Hachimi and De Thelin (J. Differ. Equ. 128:78-102, 1996) where the subcritical case was studied.
\end{abstract}

Keywords $p$-Laplace operator $\cdot$ Sub-super critical $\cdot$ Radial solution $\cdot$ Shooting method Mathematics Subject Classification 35J92 · 34B15 · 34G20

Rosa Pardo was partially supported by Grant MTM2016-75465, MINECO, Spain and Grupo de Investigación CADEDIF 920894, UCM. Sigifredo Herrón and Carlos Vélez were supported by Colciencias, Fondo Nacional de financiamiento para la ciencia, la tecnología y la innovación Francisco José de Caldas. Project Ecuaciones diferenciales dispersivas y elípticas no lineales, Code 111865842951.

$凶 \quad$ R. Pardo

rpardo@ucm.es

A. Castro

castro@g.hmc.edu

J. Cossio

jcossio@unal.edu.co

S. Herrón

sherron@unal.edu.co

C. Vélez

cauvelez@unal.edu.co

1 Department of Mathematics, Harvey Mudd College, Claremont, CA 91711, USA

2 Escuela de Matemáticas, Universidad Nacional de Colombia, Apartado Aéreo 3840, Medellín, Colombia

3 Departamento de Análisis Matemático y Matemática Aplicada, Universidad Complutense de Madrid, 28040 Madrid, Spain 


\section{Introduction}

We study the quasilinear Dirichlet problem

$$
\left\{\begin{aligned}
\Delta_{p} u+g(u)=0 & \text { in } B_{1}(0) \subset \mathbb{R}^{N}, \\
u=0 & \text { on } \partial B_{1}(0),
\end{aligned}\right.
$$

where $\triangle_{p} u=\operatorname{div}\left(|\nabla u|^{p-2} \nabla u\right), 1<p<N$, denotes the $p$-Laplacian operator. We assume that

$$
g(s):= \begin{cases}|s|^{q_{1}} & s \geq 0 \\ -|s|^{q_{2}} & s<0\end{cases}
$$

with

$$
p-1<q_{1}<p^{*}-1<q_{2}<+\infty,
$$

where $p^{*}:=\frac{N p}{N-p}$ is the critical Sobolev exponent. That is, $g$ has $p$-subcritical growth at $+\infty$ and $p$-supercritical growth at $-\infty$. Our main result is the following theorem.

Theorem 1.1 If g satisfies (1.2) and (1.3), then (1.1) has infinitely many radial solutions.

Remark 1.1 (a) Theorem 1.1 remains valid when (1.2) is replaced by a locally Lipschitz function $g$ satisfying $s g(s)>0$ for all $s \neq 0$, and either

$$
\lim _{s \rightarrow+\infty} \frac{g(s)}{s^{q_{1}}}=: L_{1} \in(0,+\infty) \text { and } \lim _{s \rightarrow-\infty} \frac{-g(s)}{|s|^{q_{2}}}=: L_{2} \in(0,+\infty),
$$

or

$$
\lim _{s \rightarrow+\infty} \frac{g(s)}{s^{q_{2}}}=: L_{3} \in(0,+\infty) \text { and } \lim _{s \rightarrow-\infty} \frac{-g(s)}{|s|^{q_{1}}}=: L_{4} \in(0,+\infty) .
$$

In the appendix (see Sect. 5), we indicate the refinements needed to extend our result for this kind of nonlinearities.

(b) Theorem 1.1 remains valid when the right-hand side in the partial differential equation in (1.1) is replaced by a continuous function of $(\|x\|, u)$ as long as such function is dominated at infinity by the nonlinearity $g$. This was done for the 2-Laplacian in [1] and for the $p$-Laplacian in [2] when $g$ is a continuous function of $\|x\|$ for subcritical problems and some sub-super critical problems. The results in [1,2] include cases where $1<p<N /(N-2)<(N+2) /(N-2)<q$ but not those where $N /(N-2)<p<$ $(N+2) /(N-2)<q$. The discussion in the appendix includes the case in which the right-hand side in (1.1) is a function of $u$.

The radial solutions to (1.1) are the solutions to

$$
\left\{\begin{aligned}
(p-1)\left|u^{\prime}\right|^{p-2} u^{\prime \prime}+\frac{N-1}{r}\left|u^{\prime}\right|^{p-2} u^{\prime}+g(u(r)) & =0, \quad 0<r<1 \\
u^{\prime}(0)=0, \quad u(1) & =0 .
\end{aligned}\right.
$$

Our proof of Theorem 1.1 is based on the analysis of the solutions to the initial value problem

$$
\left\{\begin{aligned}
(p-1)\left|u^{\prime}\right|^{p-2} u^{\prime \prime}+\frac{N-1}{r}\left|u^{\prime}\right|^{p-2} u^{\prime}+g(u(r)) & =0, \quad 0<r<1 \\
u(0)=d, \quad u^{\prime}(0) & =0 .
\end{aligned}\right.
$$

In fact, in Sect. 3, we show that the profile of the solutions of (1.7) is as shown in Fig. 1 (see below), with the number of oscillations increasing as $d \rightarrow+\infty$. 

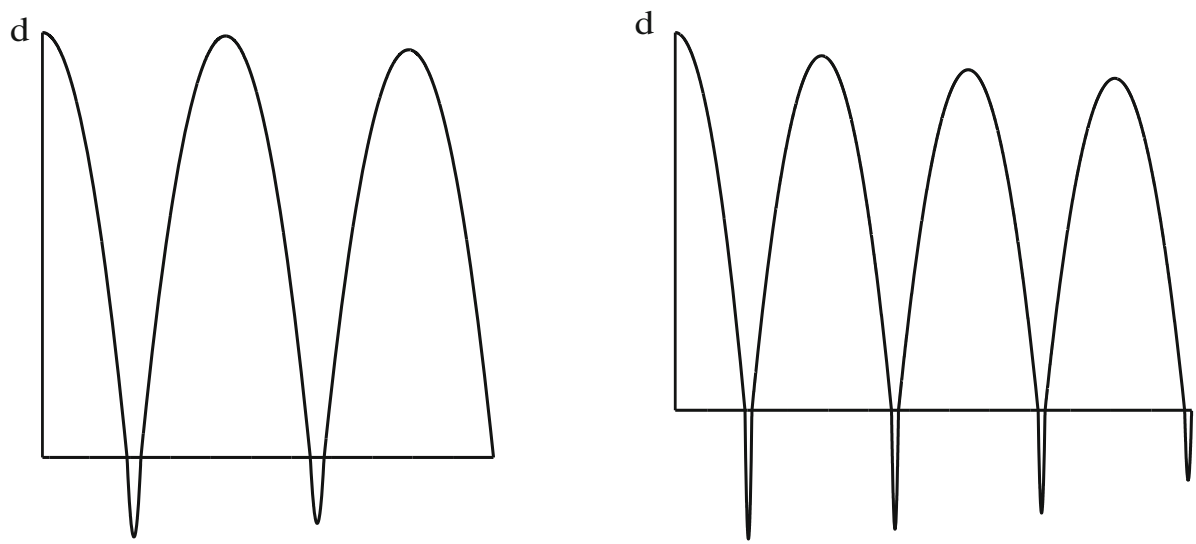

Fig. 1 Illustration of the oscillating behavior of the solutions to (1.7)

Following ideas that go back to [3], we simply prove the existence of a sequence $\left\{d_{j}\right\}_{j}$ converging to $+\infty$ such that the solution to (1.7) with $d=d_{j}$ satisfies $u(1)=0$.

Theorem 1.1 extends the results in [4] where the case $p=2$ is studied. It also extends the results in [2] where both $q_{1}, q_{2}<p^{*}$.

When $B_{1}(0)$ is replaced by an annulus, Eq. (1.6) has no singularity. This leads to the proof that (1.1) has infinitely many radial solutions, only assuming $\lim _{|s| \rightarrow \infty} g(s) /\left(s|s|^{p-2}\right)=$ $+\infty$, see [5].

We obtain $L^{\infty}$ solutions that satisfy (1.1) in the classical sense in the unit ball for $r \neq 0$, and weak solutions in the whole ball; those solutions are in $C^{1, \mu}[0,1]$ for $\mu \in(0,1)$. This is consistent with regularity theory for the $p$-Laplacian problems. Weak solutions in $W^{1, p}(\Omega) \cap L^{\infty}(\Omega)$ are in $C^{1, \mu}(\bar{\Omega})$, see [6,7].

When $p=2, q_{1}=q_{2}=(N+2) /(N-2)$ and $\Omega$ is star-shaped, $u=0$ is the only solution to (1.1), see [8]. However, if $\Omega$ has nontrivial topology then (1.1) has nontrivial solutions and it is also proved that the critical problem (1.1) has a solution in annulus $\Omega=$ $\left\{x \in \mathbb{R}^{N}: 0<a<|x|<b\right\}$, see [9].

For the $p$-Laplacian problem, it is shown that the $p$-critical problem has no positive solution in star-shaped domains, see [10]. On the other hand, if we look for radial solutions for the $p$-critical problem in annulus, it is easy to prove that (1.1) has positive solutions, see [5].

For Liouville theorems in half spaces and $1<p<N$, a priori estimates and existence of positive solutions for a more general nonlinearity $g=g(x, u, D u)$, under subcritical growth of $g$ with respect to $u$ at infinity and zero, are studied, see [11]. In [12], a priori $L^{\infty}$ bounds and existence of solutions are proved for more general nonlinearities than pure powers.

The subcritical and critical cases, both semilinear and quasilinear, have been widely studied with different techniques. See [13] for an asymptotically linear problem, [14] for a $p$-superlinear problem, [15] for bifurcation techniques in the subcritical case and [16,17] for minimax methods in critical point theory, the mountain pass theorem and variational methods in bifurcation theory. See also [18] for quasilinear equations and [19-21] and references therein for a priori bounds in general domains.

The supercritical case is not so extensively studied, see, for instance, [22-28] and references therein. For the existence and multiplicity of positive radial solutions, see [29]. See also [30,31] where the existence of infinitely many (positive) solutions to a supercritical 
problem in a ball is proved ( $p=2$ in [30], $1<p<2$ in [31]). The nonlinearities considered are linear for $u$ small and supercritical for $u$ large. Furthermore, the authors use some phase plane analysis after performing Fowler transformation.

To the best of our knowledge, the only study concerning problems with nonlinearities exhibiting a subcritical behavior at $+\infty$ and a supercritical behavior at $-\infty$ in the semilinear case is [4] (see also [1] for some related problems).

This paper is organized as follows. In Sect. 2, we introduce the energy and related functions, and their relationship. In Sect. 3, we study the oscillating qualitative properties of the solutions which will be used for proving Theorem 1.1 in Sect. 4. Finally, in the appendix, we indicate the refinements needed to extend our results to more general nonlinearities.

\section{Preliminaries and known results}

Our analysis of the solutions to the initial value problem (1.7) makes extensive use of the following quantities:

$$
\begin{aligned}
\mathcal{E}(r, d) & :=\mathcal{E}(r)=\frac{p-1}{p}\left|u^{\prime}(r)\right|^{p}+G(u(r)), \\
H(r, d) & :=r \mathcal{E}(r)+\frac{N-p}{p}\left|u^{\prime}(r)\right|^{p-2} u^{\prime}(r) u(r), \\
P(r, d) & :=\int_{0}^{r} s^{N-1}\left[N G(u(s))-\frac{N-p}{p} g(u(s)) u(s)\right] \mathrm{d} s .
\end{aligned}
$$

where $u(r)=u(r, d)$ is a solution to (1.7), and $G(s):=\int_{0}^{s} g(t) \mathrm{d} t= \begin{cases}\frac{|s|^{q_{1}+1}}{q_{1}+1}, & s \geq 0 \\ \frac{\left.|s|\right|_{2}+1}{q_{2}+1}, & s<0 .\end{cases}$

It is easy to check that for a given solution of (1.7),

$$
\mathcal{E}^{\prime}(r)=\left[(p-1)\left|u^{\prime}\right|^{p-2} u^{\prime \prime}+g(u)\right] u^{\prime}=-\frac{N-1}{r}\left|u^{\prime}\right|^{p} \leq 0, \quad \text { for } r>0,
$$

that is, the energy $\mathcal{E}$ is a decreasing function. The quantities in (2.1) are related by the Pohozaev-type identity

$$
r^{N-1} H(r, d)-t^{N-1} H(t, d)=\int_{t}^{r} s^{N-1}\left[N G(u(s))-\frac{N-p}{p} g(u(s)) u(s)\right] \mathrm{d} s,
$$

see Proposition 4.1 of [32].

Taking $t=0$ in Eq. (2.3), we have the following Pohozaev identity

$$
r^{N-1} H(r, d)=P(r, d),
$$

equivalently

$$
\begin{aligned}
r^{N} & {\left[\frac{p-1}{p}\left|u^{\prime}(r)\right|^{p}+G(u(r))\right]+\frac{N-p}{p} r^{N-1}\left|u^{\prime}(r)\right|^{p-2} u^{\prime}(r) u(r) } \\
& =\int_{0}^{r} s^{N-1}\left[N G(u(s))-\frac{N-p}{p} g(u(s)) u(s)\right] \mathrm{d} s=P(r, d) .
\end{aligned}
$$

Therefore,

$$
\text { if } u(r)=0, \quad \text { then } P(r, d)=\frac{p-1}{p} r^{N}\left|u^{\prime}(r)\right|^{p}
$$


and

$$
\text { if } u^{\prime}(r)=0, \quad \text { then } P(r, d)=r^{N} G(u(r)) .
$$

If $u(s) \geq 0, u(s) \not \equiv 0$ for all $s \in\left(t_{1}, t_{2}\right) \subset[0,1]$, then

$$
P\left(t_{2}, d\right)-P\left(t_{1}, d\right)=\frac{N-p}{p}\left[\frac{p^{*}}{q_{1}+1}-1\right] \int_{t_{1}}^{t_{2}} s^{N-1}(u(s))^{q_{1}+1} \mathrm{~d} s>0,
$$

and since the integrand is positive on $\left(t_{1}, t_{2}\right), P(r)$ is an increasing function on $\left[t_{1}, t_{2}\right]$.

On the other hand, if $u(s) \leq 0, u(s) \not \equiv 0$ for all $s \in\left(t_{1}, t_{2}\right)$, then

$$
P\left(t_{2}, d\right)-P\left(t_{1}, d\right)=-\frac{N-p}{p}\left[1-\frac{p^{*}}{q_{2}+1}\right] \int_{t_{1}}^{t_{2}} s^{N-1}|u(s)|^{q_{2}+1} \mathrm{~d} s<0,
$$

and $P(r)$ is a decreasing function on $\left[t_{1}, t_{2}\right]$.

Since (1.7) is equivalent to

$$
\left\{\begin{array}{c}
\left(r^{N-1}\left|u^{\prime}\right|^{p-2} u^{\prime}\right)^{\prime}+r^{N-1} g(u(r))=0, \quad 0<r<1 \\
u(0)=d, \quad u^{\prime}(0)=0,
\end{array}\right.
$$

integrating on $\left[t_{1}, t_{2}\right]$, we have

$$
t_{2}^{N-1}\left|u^{\prime}\left(t_{2}\right)\right|^{p-2} u^{\prime}\left(t_{2}\right)-t_{1}^{N-1}\left|u^{\prime}\left(t_{1}\right)\right|^{p-2} u^{\prime}\left(t_{1}\right)=-\int_{t_{1}}^{t_{2}} s^{N-1} g(u(s)) \mathrm{d} s .
$$

Thus, if $u(s) \geq 0, u(s) \not \equiv 0$ for all $s \in\left[t_{1}, t_{2}\right] \subset[0,1]$, then

$$
t_{2}^{N-1}\left|u^{\prime}\left(t_{2}\right)\right|^{p-2} u^{\prime}\left(t_{2}\right)-t_{1}^{N-1}\left|u^{\prime}\left(t_{1}\right)\right|^{p-2} u^{\prime}\left(t_{1}\right)=-\int_{t_{1}}^{t_{2}} s^{N-1}(u(s))^{q_{1}} \mathrm{~d} s<0 .
$$

On the other hand, if $u(s) \leq 0, u(s) \not \equiv 0$ for all $s \in\left[t_{1}, t_{2}\right]$, then

$$
t_{2}^{N-1}\left|u^{\prime}\left(t_{2}\right)\right|^{p-2} u^{\prime}\left(t_{2}\right)-t_{1}^{N-1}\left|u^{\prime}\left(t_{1}\right)\right|^{p-2} u^{\prime}\left(t_{1}\right)=\int_{t_{1}}^{t_{2}} s^{N-1}|u(s)|^{q_{2}} \mathrm{~d} s>0 .
$$

\section{Oscillation properties of the solutions to (1.7)}

Our arguments rely on the study of the oscillation behavior of solutions to (1.7). We estimate from below and from above the values of the first zero, the first point of local minimum, the second zero and the first positive point of local maximum value.

Let $u$ be a solution of (1.7), and assume that $u(0)=d>0$. Let

$$
\tau_{1}=\tau_{1}(d):=\sup \{r>0 ; u(s) \geq 0, \text { for all } s \in[0, r]\} .
$$

Thus, $\tau_{1}>0$ is the smallest zero of $u$. Let

$$
\rho_{1}=\rho_{1}(d):=\sup \left\{r>0 ; u^{\prime}(s) \leq 0 \text { for all } s \in[0, r]\right\} .
$$

That is, $\rho_{1}$ is the smallest point of minimum of $u$. Let

$$
\tau_{2}=\tau_{2}(d):=\sup \left\{r>\tau_{1}(d) ; u(s) \leq 0, \text { for all } s \in\left[\tau_{1}(d), r\right]\right\},
$$

i.e., $\tau_{2}$ is the second zero of $u$. Finally, let

$$
\sigma_{1}=\sigma_{1}(d):=\sup \left\{r>0 ; u^{\prime}(s) \geq 0, \text { for all } s \in\left[\tau_{2}(d), r\right]\right\},
$$


i.e., $\sigma_{1}$ is the first point of maximum of $u$.

By using (2.9)-(2.12), it is straightforward to check that

$$
0<\tau_{1}<\rho_{1}<\tau_{2}<\sigma_{1},
$$

and that

$$
\begin{array}{lll}
u(r)>0, & u^{\prime}(r)<0, & \text { for all } r \in\left(0, \tau_{1}\right), \\
u(r)<0, & u^{\prime}(r)<0, & \text { for all } r \in\left(\tau_{1}, \rho_{1}\right), \\
u(r)<0, & u^{\prime}(r)>0, & \text { for all } r \in\left(\rho_{1}, \tau_{2}\right), \\
u(r)>0, & u^{\prime}(r)>0, & \text { for all } \quad r \in\left(\tau_{2}, \sigma_{1}\right) .
\end{array}
$$

In Lemmas 3.2, 3.3, 3.5 and 3.6 we prove, in particular, that

$$
\left(\frac{1}{d}\right)^{\left(q_{1}+1-p\right) / p}=O\left(\tau_{1}\right)=O\left(\rho_{1}\right)=O\left(\tau_{2}\right)=O\left(\sigma_{1}\right)=O\left(\frac{1}{d}\right)^{\left(q_{1}+1-p\right) / p},
$$

for $d \gg 1$. The proof of Theorem 1.1 is based on estimating $\tau_{1}, \rho_{1}, \tau_{2}, \sigma_{1}$, and the value of $u$ at those points. We achieve this in the following four steps.

STEP 1. In this step, we prove that $\tau_{1}(d)=O\left(\left(\frac{1}{d}\right)^{\frac{q_{1}+1-p}{p}}\right)$. Let

$$
r_{1}=r_{1}(d):=\sup \{r>0 ; u(s) \geq d / 2 \text {, for all } s \in[0, r]\} .
$$

From now on, all throughout the paper, $C$ and $C_{0}, C_{1}, C_{2}, \ldots$ will denote positive constants independent of $d$ that may change from line to line.

Lemma 3.1 Let $u$ be a solution to (1.7) with $d>0$. If $r_{1}=r_{1}(d)$ is as defined by (3.8), then

(i) There exist two constants $C_{1}, C_{2}>0$, independent of $d$, such that

$$
C_{1}\left(\frac{1}{d}\right)^{\frac{q_{1}+1-p}{p}} \leq r_{1} \leq C_{2}\left(\frac{1}{d}\right)^{\frac{q_{1}+1-p}{p}} \text { for } d \gg 1 .
$$

(ii) There exists a constant $C_{3}>0$, independent of $d$, such that

$$
P\left(r_{1}, d\right) \geq C_{3} d^{N\left[1-\frac{q_{1}+1}{p^{*}}\right]} \rightarrow+\infty \quad \text { as } d \rightarrow+\infty .
$$

Proof of Lemma 3.1 (i) Let $u(0)=d>0$. Taking $\left(t_{1}, t_{2}\right)=(0, r)$ in $(2.11)$,

$$
-r^{N-1}\left|u^{\prime}(r)\right|^{p-2} u^{\prime}(r)=\int_{0}^{r} s^{N-1}(u(s))^{q_{1}} \mathrm{~d} s \geq \frac{d^{q_{1}}}{2^{q_{1}} N} r^{N}, \quad \text { for } r \in\left[0, r_{1}\right] .
$$

Hence,

$$
-u^{\prime}(r) \geq\left(\frac{d^{q_{1}}}{2^{q_{1}} N}\right)^{\frac{1}{p-1}} r^{\frac{1}{p-1}}, \quad \text { for } r \in\left[0, r_{1}\right] .
$$

Integrating on $\left[0, r_{1}\right]$, we have

$$
\frac{d}{2} \geq \frac{p-1}{p}\left(\frac{d^{q_{1}}}{2^{q_{1} N}}\right)^{\frac{1}{p-1}} r_{1}^{\frac{p}{p-1}}, \quad \text { or } \quad r_{1} \leq C_{2}\left(\frac{1}{d}\right)^{\frac{q_{1}+1-p}{p}},
$$


where $C_{2}>0$ is independent of $d$. Similarly, using that $u(r) \leq d$ for all $r \in\left[0, r_{1}\right]$, we see that there exists $C_{1}>0$, independent of $d$, such that

$$
r_{1} \geq C_{1}\left(\frac{1}{d}\right)^{\frac{q_{1}+1-p}{p}} \text { for } d>0
$$

Thus, the inequality (3.9) holds. By (1.3), $\frac{q_{1}+1}{p}>1$. This and (3.9) imply that $r_{1}(d) \rightarrow 0$ as $d \rightarrow+\infty$.

(ii) From (2.7) with $\left(t_{1}, t_{2}\right)=\left(0, r_{1}\right),(3.14)$ and (1.3), there exists a constant $C>0$ such that

$$
P\left(r_{1}, d\right) \geq C r_{1}^{N} d^{q_{1}+1} \geq C d^{N\left[1-\frac{q_{1}+1}{p^{*}}\right]} \rightarrow+\infty, \quad \text { as } d \rightarrow+\infty,
$$

which proves this lemma.

Next, we estimate the first zero $\tau_{1}(d)$ and we prove that $\tau_{1}(d)=O\left(\left(\frac{1}{d}\right)^{\frac{q_{1}+1-p}{p}}\right)$.

Lemma 3.2 If $u$ is a solution to (1.7) with $d>0$, then

(i) There exist two constants $C_{1}, C_{2}>0$, independent of $d$, such that

$$
C_{1}\left(\frac{1}{d}\right)^{\frac{q_{1}+1-p}{p}} \leq \tau_{1} \leq C_{2}\left(\frac{1}{d}\right)^{\frac{q_{1}+1-p}{p}} \text { for } d \gg 1 .
$$

(ii) There exist two constants $C_{3}, C_{4}>0$, independent of $d$, such that

$$
C_{3} d^{\frac{q_{1}+1}{p}} \leq-u^{\prime}\left(\tau_{1}\right) \leq C_{4} d^{\frac{q_{1}+1}{p}}, \quad \text { for } d \gg 1 \text {. }
$$

Remark 3.1 Observe that, from (2.4), the above lemma implies that there exist two constants $C_{5}, C_{6}>0$ such that

$$
C_{5} d^{N\left[1-\frac{q_{1}+1}{p^{*}}\right]} \leq P\left(\tau_{1}, d\right)=\frac{p-1}{p} \tau_{1}^{N}\left|u^{\prime}\left(\tau_{1}\right)\right|^{p} \leq C_{6} d^{N\left[1-\frac{q_{1}+1}{p^{*}}\right]}, \quad \text { for } d \gg 1 .
$$

Proof of Lemma 3.2 (i) Since $\tau_{1}>r_{1}$, by Lemma 3.1(i), $\tau_{1} \geq C\left(\frac{1}{d}\right)^{\frac{q_{1}+1-p}{p}}$.

From (2.11) for any $r \in\left[0, \tau_{1}\right]$,

$$
r^{N-1}\left|u^{\prime}(r)\right|^{p-1} \geq u(r)^{q_{1}} \frac{r^{N}}{N} .
$$

Hence,

$$
-u^{\prime}(r) \geq\left(\frac{1}{N}\right)^{\frac{1}{p-1}} u(r)^{\frac{q_{1}}{p-1}} r^{\frac{1}{p-1}}, \quad \forall r \in\left[0, \tau_{1}\right] .
$$

Separating variables and integrating on $\left[r_{1}, r\right]$, we have

$$
-\frac{u(r)^{1-\frac{q_{1}}{p-1}}-u\left(r_{1}\right)^{1-\frac{q_{1}}{p-1}}}{\frac{p-1-q_{1}}{p-1}} \geq\left(\frac{1}{N}\right)^{\frac{1}{p-1}} \frac{r^{\frac{p}{p-1}}-r_{1}^{\frac{p}{p-1}}}{\frac{p}{p-1}}
$$


or equivalently, for all $r \in\left[r_{1}, \tau_{1}\right]$

$$
u(r)^{1-\frac{q_{1}}{p-1}}-u\left(r_{1}\right)^{1-\frac{q_{1}}{p-1}} \geq C\left[r^{\frac{p}{p-1}}-r_{1}^{\frac{p}{p-1}}\right],
$$

where $C=C\left(p, q_{1}, N\right)=\frac{q_{1}+1-p}{p}\left(\frac{1}{N}\right)^{\frac{1}{p-1}}>0$. This and (1.3) yield

$$
u(r)^{\frac{q_{1}+1-p}{p-1}} \leq \frac{1}{C r^{\frac{p}{p-1}}\left[1-\left(\frac{r_{1}}{r}\right)^{\frac{p}{p-1}}\right]+\left(\frac{1}{u\left(r_{1}\right)}\right)^{\frac{q_{1}+1-p}{p-1}}}, \quad \forall r \in\left[r_{1}, \tau_{1}\right] .
$$

If $\tau_{1} \leq 2 r_{1}$ we are done, so let us assume $\tau_{1}>2 r_{1}$. From the preceding inequality,

$$
u(r)^{\frac{q_{1}+1-p}{p-1}} \leq \frac{1}{C r^{\frac{p}{p-1}}+\left(\frac{1}{u\left(r_{1}\right)}\right)^{\frac{q_{1}+1-p}{p-1}}}, \quad \text { for all } r \in\left[2 r_{1}, \tau_{1}\right],
$$

where $C=C\left(p, q_{1}, N\right)=\left[1-\left(\frac{1}{2}\right)^{\frac{p}{p-1}}\right] \frac{q_{1}+1-p}{p}\left(\frac{1}{N}\right)^{\frac{1}{p-1}}>0$. Thus,

$$
u(r) \leq C_{1}\left(\frac{1}{r}\right)^{\frac{p}{q_{1}+1-p}}, \quad \forall r \in\left[2 r_{1}, \tau_{1}\right], \quad \text { where } C_{1}=\left(\frac{1}{C}\right)^{\frac{p-1}{q_{1}+1-p}} .
$$

Since $P(r)$ is an increasing function on $\left[0, \tau_{1}\right]$ (see $(2.7)$ for $\left(t_{1}, t_{2}\right)=(0, r)$ with $r \in\left[0, \tau_{1}\right]$ ), from (2.4) and Lemma 3.1(ii), it follows that

$$
\begin{aligned}
P(r) & =r^{N}\left[\frac{p-1}{p}\left|u^{\prime}(r)\right|^{p}+\frac{1}{q_{1}+1}|u(r)|^{q_{1}+1}\right]+\frac{N-p}{p} r^{N-1}\left|u^{\prime}(r)\right|^{p-2} u^{\prime}(r) u(r) \\
& \geq P\left(r_{1}\right) \geq C d^{N\left[1-\frac{q_{1}+1}{p^{*}}\right], \quad \text { for all } r \in\left[r_{1}, \tau_{1}\right] .}
\end{aligned}
$$

Since $u \geq 0$ and $u^{\prime} \leq 0$ on $\left[0, \tau_{1}\right]$,

$$
r^{N}\left[\frac{p-1}{p}\left|u^{\prime}(r)\right|^{p}+\frac{1}{q_{1}+1}|u(r)|^{q_{1}+1}\right] \geq C d^{N\left[1-\frac{q_{1}+1}{p^{*}}\right]}, \quad \forall r \in\left[r_{1}, \tau_{1}\right] .
$$

If the inequality

$$
\frac{1}{q_{1}+1}|u(\hat{r})|^{q_{1}+1} \geq \frac{p-1}{p}\left|u^{\prime}(\hat{r})\right|^{p}
$$

holds true for some $\hat{r} \in\left[2 r_{1}, \tau_{1}\right]$, then, from (3.20), there exists a constant $C>0$ such that

$$
\hat{r}^{N}|u(\hat{r})|^{q_{1}+1} \geq C d^{N\left[1-\frac{q_{1}+1}{p^{*}}\right]} .
$$

This and (3.18) imply

$$
\left(\frac{1}{\hat{r}}\right)^{\frac{p\left(q_{1}+1\right)}{q_{1}+1-p}-N} \geq C d^{N\left[1-\frac{q_{1}+1}{p^{*}}\right]}
$$

Since $q_{1}$ is subcritical,

$$
\frac{p\left(q_{1}+1\right)}{q_{1}+1-p}-N=\frac{N p}{q_{1}+1-p}\left[1-\frac{q_{1}+1}{p^{*}}\right]>0 .
$$


Therefore, there exists a constant $C_{0}>0$ such that

$$
\hat{r} \leq C_{0}\left(\frac{1}{d}\right)^{\frac{q_{1}+1-p}{p}} .
$$

Let $\tilde{r}:=C_{0} M\left(\frac{1}{d}\right)^{\frac{q_{1}+1-p}{p}}$ for some constant $M>1$ such that

$$
M>3 C_{2} / C_{0},
$$

where $C_{2}$ is the constant in (3.9). If $\tau_{1} \leq \tilde{r}$, then we are done. So, we can assume that $\tau_{1}>\tilde{r}$. From (3.26), $\tilde{r} \geq 2 r_{1}$ (if $\tilde{r}<2 r_{1}$ then $M<3 C_{2} / C_{0}$, which is a contradiction). Thus, $\tau_{1}>2 r_{1}$ and (3.21) is false for all $r \in\left[\hat{r}, \tau_{1}\right]$, and then,

$$
\frac{1}{q_{1}+1}|u(r)|^{q_{1}+1} \leq \frac{p-1}{p}\left|u^{\prime}(r)\right|^{p} \quad \forall r \in\left(\tilde{r}, \tau_{1}\right) .
$$

This and (3.20) show that there exists $K>0$ such that

$$
r^{N}\left|u^{\prime}(r)\right|^{p} \geq K d^{N\left[1-\frac{q_{1}+1}{p^{*}}\right]}, \quad \forall r \in\left[\tilde{r}, \tau_{1}\right] .
$$

Since $q_{1}$ is subcritical, see (3.24), $\frac{p}{q_{1}+1-p}>\frac{N}{q_{1}+1}>\frac{N-p}{p}$. Let $M \gg 1$ be a constant such that

$$
C_{1}\left(\frac{1}{M}\right)^{\frac{p}{q_{1}+1-p}}-\frac{p K^{1 / p}}{(N-p) C_{2}^{(N-p) / p}}\left[1-\left(\frac{1}{2}\right)^{\frac{N-p}{p}}\right]\left(\frac{1}{M}\right)^{\frac{N-p}{p}}<0,
$$

where $C_{1}$ is as in (3.18), $C_{2}$ as in (3.25) and $K$ as in (3.28).

Suppose further that $\tau_{1}>2 M \tilde{r}$. From (3.28),

$$
-u^{\prime}(r) \geq K^{1 / p} d^{\frac{N}{p}\left[1-\frac{q_{1}+1}{p^{*}}\right]}\left(\frac{1}{r}\right)^{\frac{N}{p}}, \quad \forall r \in\left[M \tilde{r}, \tau_{1}\right] .
$$

Integrating on $[M \tilde{r}, r]$ for $r \leq \tau_{1}$, we have

$$
u(M \tilde{r})-u(r) \geq-\frac{p K^{1 / p}}{N-p} d^{\frac{N}{p}\left[1-\frac{q_{1}+1}{p^{*}}\right]}\left[\left(\frac{1}{r}\right)^{\frac{N}{p}-1}-\left(\frac{1}{M \tilde{r}}\right)^{\frac{N}{p}-1}\right], \quad \forall r \in\left[M \tilde{r}, \tau_{1}\right] .
$$

Therefore,

$$
u(r) \leq u(M \tilde{r})+C d^{\frac{N}{p}\left[1-\frac{q_{1}+1}{p^{*}}\right]}\left(\frac{1}{M \tilde{r}}\right)^{\frac{N}{p}-1}\left[\left(\frac{M \tilde{r}}{r}\right)^{\frac{N}{p}-1}-1\right], \quad \forall r \in\left[M \tilde{r}, \tau_{1}\right],
$$

where

$$
C:=\frac{p K^{1 / p}}{N-p}
$$

Moreover, from (3.18)

$$
d^{\frac{N}{p}\left[1-\frac{q_{1}+1}{p^{*}}\right]}\left(\frac{1}{M \tilde{r}}\right)^{\frac{N}{p}-1}=\left(\frac{1}{C_{2} M}\right)^{\frac{N-p}{p}} d^{\frac{N}{p}\left[1-\frac{q_{1}+1}{p^{*}}\right]+\frac{\left(q_{1}+1-p\right)}{p} \frac{(N-p)}{p}},
$$

and

$$
\frac{N}{p}\left[1-\frac{q_{1}+1}{p^{*}}\right]+\frac{\left(q_{1}+1-p\right)}{p} \frac{(N-p)}{p}=1 .
$$


Substituting inequality (3.18) in the first term of (3.30), (3.32) and (3.33) in the second term, by (3.29) and (3.31), we conclude that for all $r \in\left[2 M \tilde{r}, \tau_{1}\right]$

$$
\begin{aligned}
0 \leq u(r) & \leq C_{1}\left(\frac{1}{M \tilde{r}}\right)^{\frac{p}{q_{1}+1-p}}+C\left(\frac{1}{C_{2} M}\right)^{\frac{N-p}{p}}\left[\left(\frac{M \tilde{r}}{r}\right)^{\frac{N-p}{p}}-1\right] d \\
& \leq\left[C_{1}\left(\frac{1}{M}\right)^{\frac{p}{q_{1}+1-p}}-\frac{C}{C_{2}^{\frac{N-p}{p}}}\left[1-\left(\frac{1}{2}\right)^{\frac{N-p}{p}}\right]\left(\frac{1}{M}\right)^{\frac{N-p}{p}}\right] d<0 .
\end{aligned}
$$

This contradiction proves that $\tau_{1} \leq \max \left\{2 r_{1}, 2 M \tilde{r}\right\} \leq C\left(\frac{1}{d}\right)^{\frac{q_{1}+1-p}{p}}$. Thus, part (i) of the lemma is proven.

(ii) From (2.5) and (3.19) for $r=\tau_{1}$, we may write

$$
P\left(\tau_{1}, d\right)=\frac{p-1}{p} \tau_{1}^{N}\left|u^{\prime}\left(\tau_{1}\right)\right|^{p} \geq P\left(r_{1}, d\right) \geq C d^{N\left[1-\frac{q_{1}+1}{p^{*}}\right]} .
$$

This and (3.16) imply

$$
-u^{\prime}\left(\tau_{1}\right) \geq C d^{\frac{N\left(q_{1}+1\right)}{p}\left[\frac{1}{p}-\frac{1}{p^{*}}\right]}=C d^{\frac{q_{1}+1}{p}} .
$$

On the other hand, from (2.5) and (2.7) for $\left(t_{1}, t_{2}\right)=\left(0, \tau_{1}\right)$, we may write

$$
P\left(\tau_{1}, d\right)=\frac{p-1}{p} \tau_{1}^{N}\left|u^{\prime}\left(\tau_{1}\right)\right|^{p} \leq\left[\frac{1}{q_{1}+1}-\frac{1}{p^{*}}\right] d^{q_{1}+1} \tau_{1}^{N},
$$

and therefore,

$$
\left|u^{\prime}\left(\tau_{1}\right)\right|^{p} \leq \frac{p}{p-1}\left[\frac{1}{q_{1}+1}-\frac{1}{p^{*}}\right] d^{q_{1}+1} .
$$

Consequently, $\left|u^{\prime}\left(\tau_{1}\right)\right| \leq C d^{\frac{q_{1}+1}{p}}$, with $C:=\left[\frac{p}{p-1}\left(\frac{1}{q_{1}+1}-\frac{1}{p^{*}}\right)\right]^{\frac{1}{p}}$, ending the proof.

Remark 3.2 If we restrict our attention to the case $g(s)=|s|^{q_{1}}$ for $s \geq 0$ and $g(s)=-|s|^{q_{2}}$ for $s<0$, a much simpler proof of Lemma 3.2 can be made using scaling invariance: Indeed, let us denote by $u_{d}(r)$ the solution of the initial value problem (1.7), so in particular $u_{1}(r)$ denotes the solution of (1.7) for $d=1, r>0$. And let us denote by $\tau_{1}(d)$ the first zero of $u_{d}$, so in particular $\tau_{1}(1)$ denotes the first zero of $u_{1}$. One can verify that for any $d>0$, $u_{d}(r)=d u_{1}\left(r d^{\left(q_{1}+1-p\right) / p}\right)$, when $0<r<\tau_{1}(1) d^{-\left(q_{1}+1-p\right) / p}$. So, it is easy to see that the first zero of $u_{d}(r)$ satisfies $\tau_{1}(d)=\tau_{1}(1) d^{-\left(q_{1}+1-p\right) / p}$. This proves (i) in Lemma 3.2 with $C_{1}=C_{2}=\tau_{1}(1)$. Differentiating the expression for $u_{d}$ and evaluating at $r=\tau_{1}(d)$, we get (ii) in Lemma 3.2 with $C_{3}=C_{4}=-u_{1}^{\prime}\left(\tau_{1}(1)\right)$. More in general, if $u(r)$ is a positive solution when $0<r<\tau_{1}$, then $v(r)=d u\left(r d^{\left(q_{1}+1-p\right) / p}\right)$ is a solution for $d>0,0<r<$ $\tau_{1} d^{-\left(q_{1}+1-p\right) / p}$ and if $u(r)$ is negative when $\tau_{1}<r<\tau_{2}$, then $v(r)=d u_{1}\left(r d^{\left(q_{2}+1-p\right) / p}\right)$ is a solution for $d>0$ and $\tau_{1} d^{-\left(q_{2}+1-p\right) / p}<r<\tau_{2} d^{-\left(q_{2}+1-p\right) / p}$.

However, this scale invariance argument cannot be applied to more general nonlinearities as described in Remark 1.1.

STEP 2. Now, we estimate the first point of minimum $\rho_{1}(d)$. 
In the following lemma, we prove that $\rho_{1}(d)=O\left(\left(\frac{1}{d}\right)^{\frac{q_{1}+1-p}{p}}\right)$. Notice that, again, this will imply $O\left(\tau_{1}(d)\right)=O\left(\rho_{1}(d)\right)$.

Lemma 3.3 Let $u$ be a solution to (1.7) with $d>0$, if $\rho_{1}=\rho_{1}(d)$ is as defined by (3.2), then

(i) There exist two constants $C_{1}, C_{2}>0$, independent of $d$, such that

$$
C_{1}\left(\frac{1}{d}\right)^{\frac{q_{1}+1-p}{p}} \leq \rho_{1} \leq C_{2}\left(\frac{1}{d}\right)^{\frac{q_{1}+1-p}{p}} \text { for } d \gg 1 .
$$

(ii) There exist two constants $C_{3}, C_{4}>0$, independent of $d$, such that

$$
C_{3} d^{\frac{q_{1}+1}{q_{2}+1}} \leq-u\left(\rho_{1}\right) \leq C_{4} d^{\frac{q_{1}+1}{q_{2}+1}}, \text { for } d \gg 1 .
$$

(iii) There exists a constant $C_{5}>0$, independent of $d$, such that

$$
\rho_{1}-\tau_{1} \leq C_{5}\left(\frac{1}{d}\right)^{\left(q_{1}+1\right)\left[\frac{1}{p}-\frac{1}{q_{2}+1}\right]}, \quad \text { for } d \gg 1
$$

Remark 3.3 Observe that, from definition of $P$, the above lemma implies that there exist two constants $C_{6}, C_{7}>0$ such that for $d \gg 1$,

$$
C_{6} d^{N\left[1-\frac{q_{1}+1}{p^{*}}\right]} \leq P\left(\rho_{1}, d\right)=\rho_{1}^{N} G\left(u\left(\rho_{1}\right)\right)=\rho_{1}^{N} \frac{\left|u\left(\rho_{1}\right)\right|^{q_{2}+1}}{q_{2}+1} \leq C_{7} d^{N\left[1-\frac{q_{1}+1}{p^{*}}\right]}
$$

Proof of Lemma 3.3 (i) From the definition of $\rho_{1}(d)$, we have

$$
u(r) \leq 0 \quad \text { and } \quad u^{\prime}(r) \leq 0, \quad \forall r \in\left[\tau_{1}(d), \rho_{1}(d)\right] .
$$

Integrating (2.9) on $\left[\tau_{1}, r\right]$ for any $r \in\left[\tau_{1}, \rho_{1}\right]$, we may write

$$
-r^{N-1}\left|u^{\prime}(r)\right|^{p-2} u^{\prime}(r)=-\tau_{1}^{N-1}\left|u^{\prime}\left(\tau_{1}\right)\right|^{p-2} u^{\prime}\left(\tau_{1}\right)+\int_{\tau_{1}}^{r} s^{N-1}|u(s)|^{q_{2}-1} u(s) \mathrm{d} s
$$

or, equivalently,

$$
r^{N-1}\left|u^{\prime}(r)\right|^{p-1}=\tau_{1}^{N-1}\left|u^{\prime}\left(\tau_{1}\right)\right|^{p-1}-\int_{\tau_{1}}^{r} s^{N-1}|u(s)|^{q_{2}} \mathrm{~d} s .
$$

Assuming that $\rho_{1}(d) \geq 3 \tau_{1}(d)$ and

$$
\int_{\tau_{1}}^{2 \tau_{1}} s^{N-1}|u(s)|^{q 2} \mathrm{~d} s>\frac{1}{2} \tau_{1}^{N-1}\left|u^{\prime}\left(\tau_{1}\right)\right|^{p-1},
$$

we have

$$
\begin{aligned}
\int_{2 \tau_{1}}^{3 \tau_{1}} s^{N-1}|u(s)|^{q_{2}} \mathrm{~d} s & \geq\left(2 \tau_{1}\right)^{N-1} \int_{2 \tau_{1}}^{3 \tau_{1}}|u(s)|^{q_{2}} \mathrm{~d} s \\
& \geq\left(2 \tau_{1}\right)^{N-1} \tau_{1}\left|u\left(2 \tau_{1}\right)\right|^{q_{2}} \\
& \geq \int_{\tau_{1}}^{2 \tau_{1}} s^{N-1}|u(s)|^{q_{2}} \mathrm{~d} s .
\end{aligned}
$$


Thus,

$$
\int_{\tau_{1}}^{3 \tau_{1}} s^{N-1}|u(s)|^{q 2} \mathrm{~d} s>\tau_{1}^{N-1}\left|u^{\prime}\left(\tau_{1}\right)\right|^{p-1} .
$$

This and (3.43) imply

$$
-\left(3 \tau_{1}\right)^{N-1}\left|u^{\prime}\left(3 \tau_{1}\right)\right|^{p-2} u^{\prime}\left(3 \tau_{1}\right)<0,
$$

which contradicts that $\rho_{1}(d) \geq 3 \tau_{1}(d)$. Thus, if $\rho_{1}(d) \geq 3 \tau_{1}(d)$ then

$$
\int_{\tau_{1}}^{2 \tau_{1}} s^{N-1}|u(s)|^{q_{2}} \mathrm{~d} s \leq \frac{1}{2} \tau_{1}^{N-1}\left|u^{\prime}\left(\tau_{1}\right)\right|^{p-1} .
$$

This and (3.43) give

$$
-u^{\prime}(r) \geq\left(\frac{1}{2}\right)^{\frac{1}{p-1}}\left(\frac{\tau_{1}}{r}\right)^{\frac{N-1}{p-1}}\left|u^{\prime}\left(\tau_{1}\right)\right|, \quad \forall r \in\left[\tau_{1}, 2 \tau_{1}\right] .
$$

Integrating on $\left[\tau_{1}, r\right]$, for $r \in\left[\tau_{1}, 2 \tau_{1}\right]$,

$$
-u(r) \geq \frac{p-1}{N-p}\left(\frac{1}{2}\right)^{\frac{1}{p-1}}\left(\tau_{1}\right)^{\frac{N-1}{p-1}}\left|u^{\prime}\left(\tau_{1}\right)\right|\left[\left(\frac{1}{\tau_{1}}\right)^{\frac{N-p}{p-1}}-\left(\frac{1}{r}\right)^{\frac{N-p}{p-1}}\right] .
$$

From (2.7),

$$
\begin{aligned}
P(r, d)= & \frac{N}{q_{1}+1}\left[1-\frac{q_{1}+1}{p^{*}}\right] \int_{0}^{\tau_{1}} s^{N-1}|u(s)|^{q_{1}+1} \mathrm{~d} s \\
& -\frac{N}{q_{2}+1}\left[\frac{q_{2}+1}{p^{*}}-1\right] \int_{\tau_{1}}^{r} s^{N-1}|u(s)|^{q_{2}+1} \mathrm{~d} s .
\end{aligned}
$$

Since $q_{2}+1>p^{*}$

$$
\frac{1}{q_{2}+1} r^{N}|u(r)|^{q_{2}+1} \leq P(r, d) \leq P\left(\tau_{1}, d\right)=\frac{p-1}{p} \tau_{1}^{N}\left|u^{\prime}\left(\tau_{1}\right)\right|^{p}, \quad \forall r \in\left[\tau_{1}, \rho_{1}\right],
$$

see also (3.41). Therefore,

$$
|u(r)| \leq C\left(\frac{\tau_{1}}{r}\right)^{\frac{N}{q_{2}+1}}\left|u^{\prime}\left(\tau_{1}\right)\right|^{\frac{p}{q_{2}+1}}, \quad \forall r \in\left[\tau_{1}, \rho_{1}\right] .
$$

Integrating (3.47),

$$
|u(r)| \geq C \tau_{1}\left|u^{\prime}\left(\tau_{1}\right)\right|, \quad \forall r \in\left[\frac{3}{2} \tau_{1}, 2 \tau_{1}\right] .
$$

This and (3.51) yield

$$
\tau_{1}\left|u^{\prime}\left(\tau_{1}\right)\right|^{1-\frac{p}{q_{2}+1}} \leq C\left(\frac{\tau_{1}}{r}\right)^{\frac{N}{q_{2}+1}}, \quad \forall r \in\left[\frac{3}{2} \tau_{1}, 2 \tau_{1}\right] .
$$

These and $\frac{p}{N}<\frac{q_{2}+1-p}{q_{2}+1}$ give

$$
\tau_{1}\left|u^{\prime}\left(\tau_{1}\right)\right|^{\frac{p}{N}} \leq \tau_{1}\left|u^{\prime}\left(\tau_{1}\right)\right|^{1-\frac{p}{q_{2}+1}} \leq C\left(\frac{\tau_{1}}{r}\right)^{\frac{N}{q_{2}+1}} \leq C, \quad \forall r \in\left[\frac{3}{2} \tau_{1}, 2 \tau_{1}\right] .
$$


From $P\left(\tau_{1}, d\right) \geq P\left(r_{1}, d\right),(3.52)$ and (3.50), we conclude that $P\left(r_{1}, d\right)$ is bounded. Since this contradicts (3.10), we have

$$
\tau_{1} \leq \rho_{1} \leq 3 \tau_{1}, \quad \text { for } \quad d \gg 1 .
$$

(ii) From (2.4), and (2.8) for $\left(t_{1}, t_{2}\right)=\left(\tau_{1}, \rho_{1}\right)$,

$$
\rho_{1}^{N} \frac{\left|u\left(\rho_{1}\right)\right|^{q_{2}+1}}{q_{2}+1}=P\left(\rho_{1}, d\right) \leq P\left(\tau_{1}, d\right)=\frac{p-1}{p} \tau_{1}^{N}\left|u^{\prime}\left(\tau_{1}\right)\right|^{p} .
$$

This, (3.37), and Lemma 3.2(ii) imply

$$
\left|u\left(\rho_{1}\right)\right| \leq C d^{\frac{q_{1}+1}{q_{2}+1}},
$$

for some constant $C>0$ independent of $d$.

On the other hand,

$$
\begin{aligned}
P\left(\rho_{1}, d\right) & =\rho_{1}^{N} \frac{\left|u\left(\rho_{1}\right)\right|^{q_{2}+1}}{q_{2}+1} \\
& \geq P\left(\tau_{1}, d\right)-\left[\frac{1}{p^{*}}-\frac{1}{q_{2}+1}\right]\left|u\left(\rho_{1}\right)\right|^{q_{2}+1}\left(\rho_{1}^{N}-\tau_{1}^{N}\right) .
\end{aligned}
$$

Rearranging terms and dividing by $\tau_{1}^{N}$

$$
\left|u\left(\rho_{1}\right)\right|^{q_{2}+1}\left[\frac{1}{p^{*}}\left(\frac{\rho_{1}}{\tau_{1}}\right)^{N}-\left(\frac{1}{p^{*}}-\frac{1}{q_{2}+1}\right)\right] \geq \frac{p-1}{p}\left|u^{\prime}\left(\tau_{1}\right)\right|^{p} .
$$

Since (3.16), (3.37), and Lemma 3.2(ii),

$$
\left|u\left(\rho_{1}\right)\right| \geq C d^{\frac{q_{1}+1}{q_{2}+1}},
$$

for some constant $C>0$ independent of $d$, ending this part of the proof.

(iii) Let $r_{2}=r_{2}(d)$ be defined by

$$
r_{2}=r_{2}(d):=\inf \left\{r>\tau_{1} ; u(s) \leq \frac{1}{2} u\left(\rho_{1}\right), \text { for all } s \in\left[r, \rho_{1}\right]\right\} .
$$

Let $r \in\left[r_{2}, \rho_{1}\right]$. Integrating (2.9) on $\left[r, \rho_{1}\right]$,

$$
-\left|u^{\prime}(r)\right|^{p-2} u^{\prime}(r)=-\int_{r}^{\rho_{1}}\left(\frac{s}{r}\right)^{N-1} g(u(s)) \mathrm{d} s \geq \frac{\left|u\left(\rho_{1}\right)\right|^{q_{2}}}{2^{q_{2}}}\left(\rho_{1}-r\right),
$$

and therefore, $u^{\prime}(r) \leq 0$ and

$$
-u^{\prime}(r)=\left|u^{\prime}(r)\right| \geq C\left|u\left(\rho_{1}\right)\right|^{\frac{q_{2}}{p-1}}\left(\rho_{1}-r\right)^{\frac{1}{p-1}} .
$$

Integrating on $\left[r_{2}, \rho_{1}\right]$,

$$
\frac{\left|u\left(\rho_{1}\right)\right|}{2}=u\left(r_{2}\right)-u\left(\rho_{1}\right) \geq C\left|u\left(\rho_{1}\right)\right|^{\frac{q_{2}}{p-1}}\left(\rho_{1}-r_{2}\right)^{\frac{p}{p-1}},
$$

and so, from part (ii),

$$
\rho_{1}-r_{2} \leq C\left(\frac{1}{\left|u\left(\rho_{1}\right)\right|}\right)^{\frac{q_{2}+1-p}{p}} \leq C\left(\frac{1}{d}\right)^{\frac{q_{1}+1}{q_{2}+1} \frac{q_{2}+1-p}{p}} .
$$


Since $u$ is convex and decreases in $\left[\tau_{1}, \rho_{1}\right], r_{2}-\tau_{1} \leq\left(\rho_{1}-\tau_{1}\right) / 2$. This and (3.58) imply

$$
\rho_{1}-\tau_{1} \leq 2\left(\rho_{1}-r_{2}\right) \leq C\left(\frac{1}{d}\right)^{\frac{q_{1}+1}{q_{2}+1} \frac{q_{2}+1-p}{p}},
$$

which concludes the proof of Lemma.

STEP 3. Estimating the second zero $\tau_{2}(d)$. Let $r_{2}^{\prime}=r_{2}^{\prime}(d)$ be defined by

$$
r_{2}^{\prime}=r_{2}^{\prime}(d):=\sup \left\{r>\rho_{1} ; u(s) \leq \frac{1}{2} u\left(\rho_{1}\right), \text { for all } s \in\left[\rho_{1}, r\right]\right\} .
$$

In the following lemma, we first estimate $r_{2}^{\prime}=r_{2}^{\prime}(d)$.

Lemma 3.4 Let $u$ be a solution to (1.7) with $d \gg 1$. If $r_{2}^{\prime}=r_{2}^{\prime}(d)$ is as defined by (3.60), then there exists a constant $C_{3}>0$, independent of $d$, such that

$$
r_{2}^{\prime}-\rho_{1} \leq C_{3}\left(\frac{1}{d}\right)^{\left(q_{1}+1\right)\left[\frac{1}{p}-\frac{1}{q_{2}+1}\right]}, \quad \text { for } d \gg 1 .
$$

Proof of Lemma 3.4 Let $r \in\left[\rho_{1}, r_{2}^{\prime}\right]$. Thus,

$$
\left(u^{\prime}(r)\right)^{p-1}=-\int_{\rho_{1}}^{r}\left(\frac{s}{r}\right)^{N-1} g(u(s)) \mathrm{d} s \geq \frac{\left|u\left(\rho_{1}\right)\right|^{q_{2}}}{2^{q_{2}} C_{0}^{N-1}}\left(r-\rho_{1}\right) .
$$

Therefore,

$$
\left(u^{\prime}(r)\right) \geq C\left|u\left(\rho_{1}\right)\right|^{\frac{q_{2}}{p-1}}\left(r-\rho_{1}\right)^{\frac{1}{p-1}} .
$$

Integrating on $\left[\rho_{1}, r_{2}^{\prime}\right]$, we have

$$
\frac{\left|u\left(\rho_{1}\right)\right|}{2}=u\left(r_{2}^{\prime}\right)-u\left(\rho_{1}\right) \geq C\left|u\left(\rho_{1}\right)\right|^{\frac{q_{2}}{p-1}}\left[r_{2}^{\prime}-\rho_{1}\right]^{\frac{p}{p-1}} .
$$

This and (3.38) give

$$
r_{2}^{\prime}-\rho_{1} \leq C\left(\frac{1}{\left|u\left(\rho_{1}\right)\right|}\right)^{\frac{q_{2}+1-p}{p}} \leq C\left(\frac{1}{d}\right)^{\frac{q_{1}+1}{q_{2}+1} \frac{q_{2}+1-p}{p}},
$$

which proves the Lemma.

In the following lemma, we estimate the second zero $\tau_{2}(d)$ and its distance to $r_{2}^{\prime}=r_{2}^{\prime}(d)$, see (3.60).

Lemma 3.5 Let $u$ be a solution to (1.7) withd $>0$. Let $\tau_{2}=\tau_{2}(d)$ be the second zero defined by (3.3). Then,

(i) There exists a constant $C_{1}>0$, independent of $d$, such that

$$
\tau_{2}-r_{2}^{\prime} \leq C_{1}\left(\frac{1}{d}\right)^{\left(q_{1}+1\right)\left[\frac{1}{p}-\frac{1}{q_{2}+1}\right]}, \quad \text { for } d \gg 1 .
$$

(ii) There exists a constant $C_{2}>0$, independent of $d$, such that

$$
P\left(\tau_{2}, d\right) \geq C_{2} d^{N\left[1-\frac{q_{1}+1}{p^{*}}\right]}, \quad \text { for } d \gg 1 \text {. }
$$


Remark 3.4 Let us observe that by (3.61), (3.63), and Lemma 3.3(i) there exists a constant $C_{3}>0$, independent of $d$, such that

$$
\tau_{2} \leq C_{3}\left(\frac{1}{d}\right)^{\frac{q_{1}+1-p}{p}} \text { for } d \gg 1 .
$$

We note that (3.61) and (3.63) imply that there exists a constant $C_{4}>0$, independent of $d$, such that

$$
\tau_{2}-\rho_{1} \leq C_{4}\left(\frac{1}{d}\right)^{\left(q_{1}+1\right)\left[\frac{1}{p}-\frac{1}{q_{2}+1}\right]}, \quad \text { for } d \gg 1 .
$$

Also, (3.39) and (3.66) imply that there exists a constant $C_{5}>0$, independent of $d$, such that

$$
\tau_{2}-\tau_{1} \leq C_{5}\left(\frac{1}{d}\right)^{\left(q_{1}+1\right)\left[\frac{1}{p}-\frac{1}{q_{2}+1}\right]}, \quad \text { for } d \gg 1 .
$$

Proof of Lemma 3.5 (i). We argue as in the proof of part (iii) of Lemma 3.3. By the mean value theorem, (3.38) and (3.61), there exists $z \in\left(\rho_{1}, r_{2}^{\prime}\right)$, such that

$$
u^{\prime}(z)=\frac{u\left(r_{2}^{\prime}\right)-u\left(\rho_{1}\right)}{r_{2}^{\prime}-\rho_{1}}=\frac{\frac{u\left(\rho_{1}\right)}{2}-u\left(\rho_{1}\right)}{r_{2}^{\prime}-\rho_{1}}=\frac{1}{2} \frac{\left|u\left(\rho_{1}\right)\right|}{r_{2}^{\prime}-\rho_{1}} \geq C d^{\frac{q_{1}+1}{p}} .
$$

Suppose that $u(s) \leq 0$ for all $s \in[z, r]$ with $r \in[z, 2 z]$. Integrating Eq. (2.9) on $[z, s]$ and using that $g(u)<0$ for all $u<0$, we have

$$
s^{N-1}\left(u^{\prime}(s)\right)^{p-1} \geq z^{N-1}\left(u^{\prime}(z)\right)^{p-1},
$$

for all $s \in[z, r]$. Hence, by (3.68)

$$
u^{\prime}(r) \geq\left(\frac{z}{r}\right)^{\frac{N-1}{p-1}} u^{\prime}(z) \geq\left(\frac{1}{2}\right)^{\frac{N-1}{p-1}} C d^{\frac{q_{1}+1}{p}} \geq C_{1} d^{\frac{q_{1}+1}{p}} .
$$

Integrating on $[z, r]$,

$$
0 \geq u(r) \geq u(z)+C_{0} d^{\frac{q_{1}+1}{p}}(r-z) .
$$

Thus, $-u(z) \geq C_{0} d^{\frac{q_{1}+1}{p}}(r-z)$. Since $z \in\left(\rho_{1}, r_{2}^{\prime}\right),-u(z) \in\left(\left|u\left(\rho_{1}\right)\right| / 2,\left|u\left(\rho_{1}\right)\right|\right)$. This and (3.38) give

$$
C_{1} d^{\frac{q_{1}+1}{q_{2}+1}} \geq\left|u\left(\rho_{1}\right)\right| \geq|u(z)| \geq C_{0} d^{\frac{q_{1}+1}{p}}(r-z) .
$$

Hence,

$$
(r-z) \leq \frac{C_{1}}{C_{0}} d^{\frac{q_{1}+1}{q_{2}+1}-\frac{q_{1}+1}{p}}=C\left(\frac{1}{d}\right)^{\left(q_{1}+1\right)\left[\frac{1}{p}-\frac{1}{q_{2}+1}\right]}, \quad \text { for } d \gg 1 .
$$

By taking $r=2 z$ in the previous inequality, and due to $z \in\left(\rho_{1}, r_{2}^{\prime}\right)$,

$$
\rho_{1} \leq C\left(\frac{1}{d}\right)^{\left(q_{1}+1\right)\left[\frac{1}{p}-\frac{1}{q_{2}+1}\right]}, \quad \text { for } d \gg 1 .
$$

Since $\left(q_{1}+1\right)\left[\frac{1}{p}-\frac{1}{q_{2}+1}\right]>\frac{q_{1}+1-p}{p}$, this contradicts Lemma 3.3, and consequently, $u(2 z)>0$, and $\tau_{2}<2 z \leq 2 r_{2}^{\prime}$. 
Moreover, taking $r=\tau_{2}$ in (3.69),

$$
\tau_{2}-r_{2}^{\prime}<\tau_{2}-z \leq C\left(\frac{1}{d}\right)^{\left(q_{1}+1\right)\left[\frac{1}{p}-\frac{1}{q_{2}+1}\right]}, \quad \text { for } d \gg 1 .
$$

This proves part (i) of the lemma.

(ii) From definition of $P$, of $\rho_{1}$, Lemma 3.2-3.3, and (3.67)

$$
\begin{aligned}
P\left(\tau_{2}, d\right) & \geq P\left(\tau_{1}, d\right)-\left[\frac{1}{p^{*}}-\frac{1}{q_{2}+1}\right]\left|u\left(\rho_{1}\right)\right|^{q_{2}+1}\left(\tau_{2}^{N}-\tau_{1}^{N}\right) \\
& \geq P\left(\tau_{1}, d\right)-\left[\frac{1}{p^{*}}-\frac{1}{q_{2}+1}\right]\left|u\left(\rho_{1}\right)\right|^{q_{2}+1} \tau_{2}^{N-1}\left(\tau_{2}-\tau_{1}\right) \\
& \geq C_{1} d^{N\left[1-\frac{q_{1}+1}{p^{*}}\right]}-C_{2} d^{N\left[1-\frac{q_{1}+1}{p^{*}}\right]-\left(1-\frac{q_{1}+1}{q_{2}+1}\right)},
\end{aligned}
$$

where the latter inequality comes from (3.17), (3.38), (3.65), and (3.67). Due to $q_{2}>q_{1}$, part (ii) is proven and hence the lemma.

STEP 4. Estimating the first positive point of maximum, $\sigma_{1}(d)$. In the following lemma, we estimate $\sigma_{1}(d)$, defined by (3.4), and also $u\left(\sigma_{1}\right)$.

Lemma 3.6 Let $u$ be a solution to (1.7) with $d>0$. Let $\sigma_{1}=\sigma_{1}(d)$ be the first point of maximum strictly positive defined by (3.4). Then,

(i) There exist two constants $C_{1}, C_{2}>0$, independent of $d$, such that

$$
C_{1}\left(\frac{1}{d}\right)^{\frac{q_{1}+1-p}{p}} \leq \sigma_{1} \leq C_{2}\left(\frac{1}{d}\right)^{\frac{q_{1}+1-p}{p}} \text { for } d \gg 1 .
$$

(ii) There exists a constant $C_{3}>0$, independent of $d$, such that

$$
u\left(\sigma_{1}\right) \geq C_{3} d, \quad \text { for } d \gg 1 .
$$

(iii) There exists a constant $C_{4}>0$, independent of $d$, such that

$$
P\left(\sigma_{1}, d\right) \geq P\left(r_{1}, d\right) \geq C_{4} d^{N\left[1-\frac{q_{1}+1}{p^{*}}\right]}, \quad \text { for } d \gg 1 .
$$

Proof of Lemma 3.6 (i) By (3.5) and Lemma 3.3(i), $\sigma_{1}>\rho_{1} \geq C_{1}\left(\frac{1}{d}\right)^{\frac{q_{1}+1-p}{p}}$. Assuming that $p^{1 / p} u^{\prime}(r)>u^{\prime}\left(\tau_{2}\right)$ for all $r \in\left(\tau_{2}, e \tau_{2}\right)$, from (2.2), we have

$$
\begin{aligned}
\mathcal{E}\left(e \tau_{2}\right) & \leq \mathcal{E}\left(\tau_{2}\right)-\int_{\tau_{2}}^{e \tau_{2}} \frac{N-1}{p}\left|u^{\prime}\left(\tau_{2}\right)\right|^{p} \mathrm{~d} s \\
& =\frac{p-1}{p}\left|u^{\prime}\left(\tau_{2}\right)\right|^{p}-\frac{N-1}{p}\left|u^{\prime}\left(\tau_{2}\right)\right|^{p} \\
& =\frac{p-N}{p}\left|u^{\prime}\left(\tau_{2}\right)\right|^{p}<0 .
\end{aligned}
$$

Since this contradicts that $\mathcal{E}(r) \geq 0$ for all $r \in[0,1]$, we conclude that there exist $\hat{r} \in\left[\tau_{2}, e \tau_{2}\right]$ such that $u^{\prime}(\hat{r})=p^{-1 / p} u^{\prime}\left(\tau_{2}\right)$ and

$$
u^{\prime}(s) \geq p^{-1 / p} u^{\prime}\left(\tau_{2}\right) \text { for all } s \in\left[\tau_{2}, \hat{r}\right] .
$$


Integrating (2.9) on $\left[\tau_{2}, \hat{r}\right]$,

$$
\int_{\tau_{2}}^{\hat{r}} s^{N-1} u^{q_{1}}(s) \mathrm{d} s=\tau_{2}^{N-1}\left|u^{\prime}\left(\tau_{2}\right)\right|^{p-1}-\hat{r}^{N-1}\left|u^{\prime}(\hat{r})\right|^{p-1} .
$$

If $\hat{r} \leq p^{(p-1) /(2 p(N-1))} \tau_{2}$, then

$$
\begin{aligned}
\int_{\tau_{2}}^{\hat{r}} s^{N-1} u^{q_{1}}(s) \mathrm{d} s & \leq u^{q_{1}}(\hat{r}) \frac{1}{N}\left(p^{N(p-1) /(2 p(N-1))} \tau_{2}^{N}-\tau_{2}^{N}\right) \\
& =u^{q_{1}}(\hat{r}) \frac{1}{N} \tau_{2}^{N}\left(p^{N(p-1) /(2 p(N-1))}-1\right) .
\end{aligned}
$$

Now,

$$
\begin{aligned}
\tau_{2}^{N-1}\left|u^{\prime}\left(\tau_{2}\right)\right|^{p-1}-\hat{r}^{N-1}\left|u^{\prime}(\hat{r})\right|^{p-1} \geq & \tau_{2}^{N-1}\left|u^{\prime}\left(\tau_{2}\right)\right|^{p-1} \\
& -p^{(p-1) /(2 p)} \tau_{2}^{N-1} p^{-(p-1) / p}\left|u^{\prime}\left(\tau_{2}\right)\right|^{p-1} \\
\geq & \tau_{2}^{N-1}\left|u^{\prime}\left(\tau_{2}\right)\right|^{p-1}\left(1-p^{(1-p) /(2 p)}\right)
\end{aligned}
$$

From (3.74), (3.75), and (3.76),

$$
\frac{1}{N} \tau_{2}\left(p^{N(p-1) /(2 p(N-1))}-1\right) u^{q_{1}}(\hat{r}) \geq\left|u^{\prime}\left(\tau_{2}\right)\right|^{p-1}\left(1-p^{(1-p) /(2 p)}\right) .
$$

From (1.7) with $g(u)=u^{q_{1}}$, it follows that if $r \geq \hat{r}$ is such that $u^{\prime}(s)>0$ for all $s \in[\hat{r}, r]$ then $u$ is concave on $[\hat{r}, r]$. We claim that $u^{\prime \prime}(s) \leq-K\left|u^{\prime}\left(\tau_{2}\right)\right| / \tau_{2}$ where $K$ is a positive constant depending only on $p$ and $N$. In fact, due to (1.7) and (3.77),

$$
(p-1)\left(u^{\prime}(s)\right)^{p-2} u^{\prime \prime}(s)<-u^{q_{1}}(s) \leq-u^{q_{1}}(\hat{r}) \leq-C(N, p)\left(u^{\prime}\left(\tau_{2}\right)\right)^{p-1} / \tau_{2}
$$

and since $u^{\prime \prime}(s)<0$, we obtain $(p-1)\left(u^{\prime}(s)\right)^{p-2} u^{\prime \prime}(s) \geq(p-1)\left(u^{\prime}(\hat{r})\right)^{p-2} u^{\prime \prime}(s)$, provided $p \geq 2$. Combining these last two inequalities and taking into account $u^{\prime}(\hat{r})=p^{-1 / p} u^{\prime}\left(\tau_{2}\right)$ the claim follows. If $p<2$, (1.7) gives us

$$
(p-1) u^{\prime \prime}(s) \leq-u^{q_{1}}(s)\left(u^{\prime}(s)\right)^{2-p} \leq-u^{q_{1}}(\hat{r})\left(u^{\prime}(s)\right)^{2-p} .
$$

Combining (3.73), (3.78), and (3.79), the claim is obtained. Thus, integrating $u^{\prime \prime}(s) \leq$ $-K\left|u^{\prime}\left(\tau_{2}\right)\right| / \tau_{2}$ on $[\hat{r}, r]$, we have

$$
0 \leq u^{\prime}(r) \leq p^{-1 / p} u^{\prime}\left(\tau_{2}\right)-K \frac{u^{\prime}\left(\tau_{2}\right)}{\tau_{2}}(r-\hat{r}) .
$$

Assume $u^{\prime}(r)>0$ for all $r>\hat{r}$. By taking $r$ such that $r-\hat{r}>\frac{2 \tau_{2}}{K} p^{-1 / p}$, from (3.80) we have $u^{\prime}(r)<0$, which is a contradiction. This proves that there exists $\sigma_{1}>\hat{r}>\tau_{2}$ such that $u^{\prime}\left(\sigma_{1}\right)=0$ and $u^{\prime}(s)>0$ on $\left[\tau_{2}, \sigma_{1}\right]$. Moreover, from (3.80),

$$
\sigma_{1} \leq \hat{r}+\frac{\tau_{2}}{K} p^{-1 / p} \leq\left(e+\frac{p^{-1 / p}}{K}\right) \tau_{2}
$$

On the other hand, if $\hat{r} \geq p^{(p-1) /(2 p(N-1))} \tau_{2}$ and since $u^{\prime}(s) \geq p^{-1 / p} u^{\prime}\left(\tau_{2}\right)$ for all $s \in\left[\tau_{2}, \hat{r}\right]$, then integrating the last inequality on $\left[\tau_{2}, \hat{r}\right]$,

$$
\begin{aligned}
u(\hat{r}) & \geq p^{-1 / p} u^{\prime}\left(\tau_{2}\right)\left(\hat{r}-\tau_{2}\right) \geq p^{-1 / p} u^{\prime}\left(\tau_{2}\right) \tau_{2}\left(p^{(p-1) /(2 p(N-1))}-1\right) \\
& =K_{1} u^{\prime}\left(\tau_{2}\right) \tau_{2}
\end{aligned}
$$


where $K_{1}=p^{-1 / p}\left(p^{(p-1) /(2 p(N-1))}-1\right)>0$. If we assume $u^{\prime}(r)>0$ for all $r>\hat{r}$, because of (2.9), since $\hat{r} \leq e \tau_{2}$ and $u^{\prime}(\hat{r})=p^{-1 / p} u^{\prime}\left(\tau_{2}\right)$, we obtain

$$
\begin{aligned}
r^{N-1} u^{\prime}(r)^{p-1} & =\hat{r}^{N-1} u^{\prime}(\hat{r})^{p-1}-\int_{\hat{r}}^{r} s^{N-1} u(s)^{q_{1}} \mathrm{~d} s \\
& \leq \mathrm{e}^{N-1} \tau_{2}^{N-1} p^{-(p-1) / p} u^{\prime}\left(\tau_{2}\right)^{p-1}-\int_{\hat{r}}^{r} s^{N-1} u(s)^{q_{1}} \mathrm{~d} s .
\end{aligned}
$$

From (3.81), it follows

$$
\begin{aligned}
\int_{\hat{r}}^{r} s^{N-1} u(s)^{q_{1}} \mathrm{~d} s & \geq u(\hat{r})^{q_{1}} \int_{\hat{r}}^{r} s^{N-1} \geq u(\hat{r})^{q_{1}} \tau_{2}^{N-1}(r-\hat{r}) \\
& \geq K_{1}^{q_{1}} u^{\prime}\left(\tau_{2}\right)^{q_{1}} \tau_{2}^{q_{1}} \tau_{2}^{N-1}(r-\hat{r}) .
\end{aligned}
$$

From (3.82) and (3.83),

$$
0 \leq r^{N-1} u^{\prime}(r)^{p-1} \leq \tau_{2}^{N-1} u^{\prime}\left(\tau_{2}\right)^{p-1}\left[\mathrm{e}^{N-1} p^{-(p-1) / p}-K_{1}^{q_{1}} u^{\prime}\left(\tau_{2}\right)^{q_{1}-p+1} \tau_{2}^{q_{1}}(r-\hat{r})\right] .
$$

Taking $r$ such that

$$
r-\hat{r}>\frac{2 \mathrm{e}^{N-1} p^{-(p-1) / p}}{K_{1}^{q_{1}} u^{\prime}\left(\tau_{2}\right)^{q_{1}-p+1} \tau_{2}^{q_{1}}},
$$

from (3.84), we have $u^{\prime}(r)<0$, which is a contradiction. Thus, there exists $\sigma_{1}>\hat{r}>\tau_{2}$ such that $u^{\prime}\left(\sigma_{1}\right)=0$ and $u^{\prime}(s)>0$ on $\left[\tau_{2}, \sigma_{1}\right]$. Moreover, from (3.84)

$$
\sigma_{1}-\hat{r} \leq C\left(N, p, q_{1}\right) \frac{1}{u^{\prime}\left(\tau_{2}\right)^{q_{1}-p+1}} \frac{1}{\tau_{2}^{q_{1}}} .
$$

We claim that

$$
u^{\prime}\left(\tau_{2}\right) \geq C d^{\left(q_{1}+1\right) / p} .
$$

In fact, due to (2.5) and (3.64), $\frac{p-1}{p} \tau_{2}^{N}\left|u^{\prime}\left(\tau_{2}\right)\right|^{p}=P\left(\tau_{2}, d\right) \geq C_{2} d^{N\left[1-\frac{q_{1}+1}{p^{*}}\right]}$. Thus, by using (3.65),

$$
\begin{aligned}
\left|u^{\prime}\left(\tau_{2}\right)\right|^{p} & \geq C \frac{d^{N\left[1-\frac{q_{1}+1}{p^{*}}\right]}}{\tau_{2}^{N}} \geq C d^{N\left[1-\frac{q_{1}+1}{\left.p^{*}\right]}\right.} d^{\frac{N}{p}\left(q_{1}+1-p\right)} \\
& =C d^{q_{1}+1} .
\end{aligned}
$$

The claim follows. Now, from (3.85), (3.86), and (3.65),

$$
\sigma_{1}-\hat{r} \leq C\left(\frac{1}{d}\right)^{\frac{q_{1}+1}{p}\left(q_{1}+1-p\right)}\left(\frac{1}{d}\right)^{\frac{-q_{1}}{p}\left(q_{1}+1-p\right)},
$$

and then,

$$
\sigma_{1} \leq \hat{r}+C\left(\frac{1}{d}\right)^{\frac{q_{1}+1-p}{p}}
$$

Since $\hat{r} \leq e \tau_{2}$ and (3.65),

$$
\sigma_{1} \leq C\left(\frac{1}{d}\right)^{\frac{q_{1}+1-p}{p}} .
$$

This proves part (i) of the lemma. 
(ii) From the definition of $P$, see (2.6) and (2.7) for $\left(t_{1}, t_{2}\right)=\left(\tau_{2}, \sigma_{1}\right)$, and (3.64), we have

$$
\frac{\sigma_{1}^{N}\left|u\left(\sigma_{1}\right)\right|^{q_{1}+1}}{q_{1}+1}=P\left(\sigma_{1}, d\right)>P\left(\tau_{2}, d\right) \geq C d^{N\left[1-\frac{q_{1}+1}{p^{*}}\right]}, \quad \text { for } d \gg 1 .
$$

Hence,

$$
u\left(\sigma_{1}\right) \geq C d^{N\left[\frac{1}{q_{1}+1}-\frac{1}{p^{*}}\right]}\left(\frac{1}{\sigma_{1}}\right)^{\frac{N}{q_{1}+1}} \geq C d .
$$

(iii) The definition of $P$ and the nonlinearity $g$ give us

$$
\begin{aligned}
P\left(\sigma_{1}, d\right)= & P\left(r_{1}, d\right)+N\left(\frac{1}{q_{1}+1}-\frac{1}{p^{*}}\right) \int_{r_{1}}^{\tau_{1}} s^{N-1}(u(s))^{q_{1}+1} \mathrm{~d} s \\
& -N\left(\frac{1}{p^{*}}-\frac{1}{q_{2}+1}\right) \int_{\tau_{1}}^{\tau_{2}} s^{N-1}|u(s)|^{q_{2}+1} \mathrm{~d} s \\
& +N\left(\frac{1}{q_{1}+1}-\frac{1}{p^{*}}\right) \int_{\tau_{2}}^{\sigma_{1}} s^{N-1}(u(s))^{q_{1}+1} \mathrm{~d} s .
\end{aligned}
$$

Let $r_{1}^{\prime}=r_{1}^{\prime}(d):=\sup \{r>0 ; u(s) \geq d / 4$ on $[0, r]\}$. Since $\mathcal{E}$ is a nonincreasing function, see (2.2), $\left|u^{\prime}(s)\right| \leq \frac{p}{(p-1)\left(q_{1}+1\right)} d^{\left(q_{1}+1\right) / p}$ for all $s \in\left[r_{1}, r_{1}^{\prime}\right]$. Integrating $-u^{\prime}$ in the latter interval, we have $d \leq 4 \frac{p}{(p-1)\left(q_{1}+1\right)} d^{\left(q_{1}+1\right) / p}\left(r_{1}^{\prime}-r_{1}\right)$, or $r_{1}^{\prime}-r_{1} \geq C d^{\left(p-q_{1}-1\right) / p}$. Therefore,

$$
\begin{aligned}
\int_{r_{1}}^{\tau_{1}} s^{N-1}(u(s))^{q_{1}+1} \mathrm{~d} s & \geq \int_{r_{1}}^{r_{1}^{\prime}} s^{N-1}(u(s))^{q_{1}+1} \mathrm{~d} s \\
& \geq \frac{1}{N}\left(\frac{d}{4}\right)^{q_{1}+1} r_{1}^{N-1}\left[r_{1}^{\prime}-r_{1}\right] \\
& \geq \hat{C} d^{q_{1}+1-\frac{q_{1}+1-p}{p}[(N-1)+1]} \equiv \hat{C} d^{\xi},
\end{aligned}
$$

where $\xi:=N\left[1-\frac{q_{1}+1}{p^{*}}\right]$.

On the other hand, from (3.38), (3.67), and Lemma 3.5 we have

$$
\int_{\tau_{1}}^{\tau_{2}} s^{N-1}|u(s)|^{q_{2}+1} \mathrm{~d} s \leq \bar{C} d^{q_{1}+1-\frac{q_{1}+1-p}{p}(N-1)-\left(q_{1}+1\right)\left[\frac{1}{p}-\frac{1}{q_{2}+1}\right]} \equiv \bar{C} d^{\eta}
$$

where $\eta:=N\left[1-\frac{q_{1}+1}{p^{*}}\right]-\left(1-\frac{q_{1}+1}{q_{2}+1}\right)$. Since $0<\eta<\xi$, for $d$ large, the first integral in (3.87) is larger than the second. Since, in addition, the third integral in (3.87) is positive we have $P\left(\sigma_{1}, d\right) \geq P\left(r_{1}, d\right)$, which proves the lemma.

\section{Proof of Theorem 1.1}

Proof Let $d_{2}>0$ be such that if $d>d_{2}$ then

$$
\left(\frac{1}{q_{1}+1}-\frac{1}{p^{*}}\right) \hat{C} d^{\xi}-\left(\frac{1}{p^{*}}-\frac{1}{q_{2}+1}\right) \bar{C} d^{\eta}>0
$$

where $\hat{C}, \xi, \bar{C}, \eta$ are as in (3.88) and (3.89). Repeating the arguments developed in Sect. 3, we see that if $d>d_{2}$, then there exists $\sigma_{2}(d):=\sigma_{2}$ such that $u$ has a local maximum at $\sigma_{2}>\sigma_{1}$, there exists $\rho_{2} \in\left(\sigma_{1}, \sigma_{2}\right)$ such that $u^{\prime}<0$ on $\left(\sigma_{1}, \rho_{2}\right), u^{\prime}>0$ in $\left(\rho_{2}, \sigma_{2}\right)$, and 
there exist $\tau_{3} \in\left(\sigma_{1}, \rho_{2}\right)$ and $\tau_{4} \in\left(\rho_{2}, \sigma_{2}\right)$ with $u\left(\tau_{3}\right)=u\left(\tau_{4}\right)=0, \sigma_{2}=O\left(\sigma_{1}\right)$ and for $r \in\left[\sigma_{1}, \tau_{3}\right]$

$$
\begin{aligned}
P(r, d) & =P\left(\sigma_{1}, d\right)+N\left(\frac{1}{q_{1}+1}-\frac{1}{p^{*}}\right) \int_{\sigma_{1}}^{r} s^{N-1}(u(s))^{q_{1}+1} \mathrm{~d} s \\
& \geq P\left(\sigma_{1}, d\right) .
\end{aligned}
$$

Also, for $r \in\left[\tau_{3}, \sigma_{2}\right]$

$$
\begin{aligned}
P(r, d) \geq & P\left(\sigma_{1}, d\right)+N\left(\frac{1}{q_{1}+1}-\frac{1}{p^{*}}\right) \int_{\sigma_{1}}^{\tau_{3}} s^{N-1}(u(s))^{q_{1}+1} \mathrm{~d} s \\
& -N\left(\frac{1}{p^{*}}-\frac{1}{q_{2}+1}\right) \int_{\tau_{3}}^{\tau_{4}} s^{N-1}|u(s)|^{q_{2}+1} \mathrm{~d} s \\
\geq & P\left(\sigma_{1}, d\right)+N\left[\left(\frac{1}{q_{1}+1}-\frac{1}{p^{*}}\right) \hat{C} d^{\xi}-\left(\frac{1}{p^{*}}-\frac{1}{q_{2}+1}\right) \bar{C} d^{\eta}\right] \\
\geq & P\left(\sigma_{1}, d\right) .
\end{aligned}
$$

Hence, for all $r \in\left[\sigma_{1}, \sigma_{2}\right]$,

$$
P(r, d) \geq P\left(\sigma_{1}, d\right) \rightarrow \infty \quad \text { as } d \rightarrow \infty .
$$

Iterating this argument, we find $\sigma_{3}(d):=\sigma_{3}<\cdots<\sigma_{k}(d):=\sigma_{k} \leq 1<\sigma_{k+1}$ such that for each $i=2, \ldots, k+1, u$ has a maximum at $\sigma_{i}$ (recall $\left.\sigma_{0}=0\right)$, there exists $\rho_{i} \in\left(\sigma_{i-1}, \sigma_{i}\right)$ with $u^{\prime}<0$ on $\left(\sigma_{i-1}, \rho_{i}\right), u^{\prime}>0$ in $\left(\rho_{i}, \sigma_{i}\right)$, and there exist $\tau_{2 i-1} \in\left(\sigma_{i-1}, \rho_{i}\right)$ and $\tau_{2 i} \in\left(\rho_{i}, \sigma_{i}\right)$ with $u\left(\tau_{2 i-1}\right)=u\left(\tau_{2 i}\right)=0, \sigma_{i}=O\left(\sigma_{i-1}\right)$ (see (3.7)) and

$$
P(r, d) \geq P\left(\sigma_{1}, d\right) \rightarrow \infty \quad \text { as } d \rightarrow \infty
$$

for all $r \in\left[\sigma_{1}, 1\right]$, see Lemma 3.6(iii). For $r \in\left[0, \sigma_{1}\right], \mathcal{E}(r) \geq \mathcal{E}\left(\sigma_{1}\right)=G\left(u\left(\sigma_{1}\right)\right) \geq$ $C d^{q_{1}+1}$. From this inequality, (4.5), and (2.4), we may assume that $\mathcal{E}(r, d) \geq 1$ for all $r \in[0,1]$. Hence, $\left(u(r, d), u^{\prime}(r, d)\right) \neq(0,0)$ for all $r \in(0,1]$ which implies that there exists a differentiable function $\phi(r, d)$ such that $\phi(0, d)=0$,

$$
\begin{gathered}
u(r, d)=\sqrt{(u(r, d))^{2}+\left(u^{\prime}(r, d)\right)^{2}} \cos \phi(r, d), \\
u^{\prime}(r, d)=-\sqrt{(u(r, d))^{2}+\left(u^{\prime}(r, d)\right)^{2}} \sin \phi(r, d) .
\end{gathered}
$$

Suppose now that $u(T, d)=0$, i.e., $\phi(T, d)=j \pi+\pi / 2$ for some nonnegative integer $j$. Differentiating the first equation in (4.6) with respect to $r$, we have

$$
u^{\prime}(T, d)=-\sqrt{(u(T, d))^{2}+\left(u^{\prime}(T, d)\right)^{2}} \sin (\phi(T, d)) \phi^{\prime}(T, d) .
$$

Because $u(T, d)=0, u^{\prime}(T, d) \neq 0$. Thus, the second equation in (4.6) and (4.7) give $\phi^{\prime}(T, d)=1$. Hence, $\phi(t, d)>\phi(T, d)$ for all $t \in(T, 1]$. In fact, assuming to the contrary that $\phi\left(T_{1}, d\right)=\phi(T, d)$ for some $T_{1} \in(T, 1]$, then by the continuity of $\phi(\cdot, d)$ we may assume $\phi(t, d)>\phi(T, d)$ for all $t \in\left(T, T_{1}\right)\left(\operatorname{simply}\right.$ take $T_{1}=\sup \{t \in(T, 1] ; \phi(t, d)>$ $\phi(T, d)\})$. Since $\phi(t, d)>\phi\left(T_{1}, d\right)$ for $t \in\left(T, T_{1}\right), \phi^{\prime}\left(T_{1}, d\right) \leq 0$ which contradicts that $\phi^{\prime}\left(T_{1}, d\right)=1$ since $\phi\left(T_{1}, d\right)=\phi(T, d)=j \pi+\pi / 2$.

Since $u$ decreases in $\left[0, \rho_{1}\right]$ and $u^{\prime}\left(\rho_{1}\right)=0, \phi\left(\rho_{1}\right)=\pi$. Similarly, $\phi\left(\sigma_{1}\right)=2 \pi$. Iterating this, we see that $\phi\left(\sigma_{k}\right)=2 k \pi$. By the definition of $\tau_{2 k}, \phi\left(\tau_{2 k}, d\right)=2 k \pi-\pi / 2$. Hence, $\lim _{d \rightarrow+\infty} \phi(1, d)=+\infty$. This and the intermediate value theorem imply the existence of a sequence $\left\{\delta_{j}\right\} \rightarrow+\infty$ such that $\phi\left(1, \delta_{j}\right)=j \pi+\frac{\pi}{2}$, in other words $u\left(1, \delta_{j}\right)=0$. This proves the theorem. 
Acknowledgements The authors want to express their deep gratitude to the anonymous referees for their helpful comments.

\section{Appendix: On a more general nonlinearity}

In this appendix, we indicate the refinements needed to extend our results to a more general nonlinearity $g$ satisfying hypothesis (1.4) of Remark 1.1(a).

Note that under hypothesis (1.4), for every $\varepsilon>0$, there exist two constants $M_{\varepsilon}>0, M_{\varepsilon}^{\prime}>$ 0 such that

$$
\begin{aligned}
&\left(L_{1}-\varepsilon\right) s^{q_{1}}-M_{\varepsilon} \leq g(s) \leq\left(L_{1}+\varepsilon\right) s^{q_{1}}+M_{\varepsilon}, \quad \forall s>0, \\
&\left(L_{2}-\varepsilon\right)|s|^{q_{2}}-M_{\varepsilon} \leq-g(s)=|g(s)| \leq\left(L_{2}+\varepsilon\right)|s|^{q_{2}}+M_{\varepsilon}, \quad \forall s<0,
\end{aligned}
$$

and

$$
\begin{array}{ll}
\frac{\left(L_{1}-\varepsilon\right)}{q_{1}+1} s^{q_{1}+1}-M_{\varepsilon}^{\prime} \leq G(s) \leq \frac{\left(L_{1}+\varepsilon\right)}{q_{1}+1} s^{q_{1}+1}+M_{\varepsilon}^{\prime}, & \forall s>0, \\
\frac{\left(L_{2}-\varepsilon\right)}{q_{1}+1}|s|^{q_{2}+1}-M_{\varepsilon}^{\prime} \leq G(s) \leq \frac{\left(L_{2}+\varepsilon\right)}{q_{2}+1}|s|^{q_{2}+1}+M_{\varepsilon}^{\prime}, \quad \forall s<0,
\end{array}
$$

where $G(s):=\int_{0}^{s} g(t) d t$.

Moreover, for all $s>0$,

$$
\begin{aligned}
\left(\frac{p^{*}\left(L_{1}-\varepsilon\right)}{q_{1}+1}-\left(L_{1}+\varepsilon\right)\right) s^{q_{1}+1}-M_{\varepsilon}^{\prime \prime} & \leq p^{*} G(s)-s g(s) \\
& \leq\left(\frac{p^{*}\left(L_{1}+\varepsilon\right)}{q_{1}+1}-\left(L_{1}-\varepsilon\right)\right) s^{q_{1}+1}+M_{\varepsilon}^{\prime \prime},
\end{aligned}
$$

and for all $s<0$,

$$
\begin{aligned}
-\left(\left(L_{2}+\varepsilon\right)-\frac{p^{*}\left(L_{2}-\varepsilon\right)}{q_{2}+1}\right)|s|^{q_{2}+1}-M_{\varepsilon}^{\prime \prime} & \leq p^{*} G(s)-s g(s) \\
& \leq-\left(\left(L_{2}-\varepsilon\right)-\frac{p^{*}\left(L_{2}+\varepsilon\right)}{q_{2}+1}\right)|s|^{q_{2}+1}+M_{\varepsilon}^{\prime \prime} .
\end{aligned}
$$

Hence, there exist constants $C_{i}>0, i=1, \ldots, 4$ and $M>0$ such that

$$
\begin{aligned}
C_{1} s^{q_{1}+1}-M & \leq p^{*} G(s)-s g(s) \leq C_{2} s^{q_{1}+1}+M, \quad \forall s>0, \\
-C_{3}|s|^{q_{2}+1}-M & \leq p^{*} G(s)-s g(s) \leq-C_{4}|s|^{q_{2}+1}+M, \quad \forall s<0 . \\
C_{5}|s|^{q_{1}+1}-M & \leq G(s) \leq C_{6}|s|^{q_{1}+1}+M, \quad \forall s \in \mathbb{R},
\end{aligned}
$$

and

$$
\begin{gathered}
C_{7} s^{q_{1}}-M \leq g(s) \leq C_{8} s^{q_{1}}+M, \quad \forall s>0, \\
C_{9}|s|^{q_{2}}-M \leq-g(s)=|g(s)| \leq C_{10}|s|^{q_{2}}+M, \quad \forall s<0 .
\end{gathered}
$$

Furthermore, Eqs. (2.7) and (2.8) should both be substituted by

$$
P\left(t_{2}, d\right)-P\left(t_{1}, d\right)=\frac{N-p}{p} \int_{t_{1}}^{t_{2}} s^{N-1}\left[p^{*} G(u(s))-g(u(s)) u(s)\right] \mathrm{d} s,
$$


and Eqs. (2.11) and (2.12) should be substituted by

$$
\begin{aligned}
& t_{2}^{N-1}\left|u^{\prime}\left(t_{2}\right)\right|^{p-2} u^{\prime}\left(t_{2}\right)-t_{1}^{N-1}\left|u^{\prime}\left(t_{1}\right)\right|^{p-2} u^{\prime}\left(t_{1}\right)=-\int_{t_{1}}^{t_{2}} s^{N-1} g(u(s)) \mathrm{d} s<0, \\
& t_{2}^{N-1}\left|u^{\prime}\left(t_{2}\right)\right|^{p-2} u^{\prime}\left(t_{2}\right)-t_{1}^{N-1}\left|u^{\prime}\left(t_{1}\right)\right|^{p-2} u^{\prime}\left(t_{1}\right)=\int_{t_{1}}^{t_{2}} s^{N-1}|g(u(s))| \mathrm{d} s>0,
\end{aligned}
$$

respectively.

Remark 5.1 The proof of Lemma 3.1 should be modified in the following way.

Let $u(0)=d \gg 1$. Taking $\left(t_{1}, t_{2}\right)=(0, r)$ in (2.11), Eq. (3.11) in part (i) should be substituted by

$$
\begin{aligned}
-r^{N-1}\left|u^{\prime}(r)\right|^{p-2} u^{\prime}(r) & =\int_{0}^{r} s^{N-1} g(u(s)) \mathrm{d} s \geq\left(C d^{q_{1}}-M\right) r^{N} \\
& \geq \frac{C}{2} d^{q_{1}} r^{N}, \quad \text { for } r \in\left[0, r_{1}\right],
\end{aligned}
$$

and Eq. (3.15) in part (ii) should be substituted by

$$
P\left(r_{1}, d\right) \geq C r_{1}^{N}\left(d^{q_{1}+1}-M\right) \geq \frac{C}{2} d^{N\left[1-\frac{q_{1}+1}{p^{*}}\right]} \rightarrow+\infty, \quad \text { as } d \rightarrow+\infty,
$$

respectively. All the others arguments remain unchanged, except possibly the constants involved.

Remark 5.2 We now write the proof of Lemma 3.2 for a generalized nonlinearity.

(i) Since $\tau_{1}>r_{1}$, by Lemma 3.1(i), $\tau_{1} \geq C\left(\frac{1}{d}\right)^{\frac{q_{1}+1-p}{p}}$.

From (5.12) and (5.10), for any $r \in\left[0, \tau_{1}\right]$,

$$
r^{N-1}\left|u^{\prime}(r)\right|^{p-1} \geq\left(C_{7} u(r)^{q_{1}}-M\right) \frac{r^{N}}{N} .
$$

Thanks to (5.1) and (5.7), there exist positive constants $C_{7}, C_{1}, M$ such that $g(u(r)) \geq$ $C_{7} u(r)^{q_{1}}-M$ and $p^{*} G(u(r))-u(r) g(u(r)) \geq C_{1}(u(s))^{q_{1}+1}-M, \forall r \in\left[0, \tau_{1}\right]$. Let us define $r^{*}$ such that

$$
r^{*}=r^{*}(d):=\sup \left\{r>0 ; u(s) \geq \max \left\{\left(\frac{M}{C_{7}}\right)^{\frac{1}{q_{1}}},\left(\frac{M}{C_{1}}\right)^{\frac{1}{q_{1}+1}}\right\}, \forall s \in[0, r]\right\} .
$$

By definition, $r^{*} \leq \tau_{1}$. Moreover, we observe that $g(u(r)) \geq 0$ and

$$
p^{*} G(u(r))-u(r) g(u(r)) \geq 0, \forall r \in\left[0, r^{*}\right) .
$$

We claim that $r^{*} \leq C\left(\frac{1}{d}\right)^{\frac{q_{1}+1-p}{p}}$ for some $C>0$ : since

$$
x^{q}-a \geq\left(x-a^{1 / q}\right)^{q}, \quad \text { for all } x \geq a^{1 / q}, \quad q>1, a>0,
$$

we have

$$
\left|u^{\prime}(r)\right|^{p-1} \geq C_{7}\left(u(r)-\left(\frac{M}{C_{7}}\right)^{\frac{1}{q_{1}}}\right)^{q_{1}} \frac{r}{N}, \quad \forall r \in\left[0, r^{*}\right) .
$$


Hence,

$$
-u^{\prime}(r) \geq\left(\frac{C_{7}}{N}\right)^{\frac{1}{p-1}}\left(u(r)-\left(\frac{M}{C_{7}}\right)^{\frac{1}{q_{1}}}\right)^{\frac{q_{1}}{p-1}} r^{\frac{1}{p-1}}, \quad \forall r \in\left[0, r^{*}\right) .
$$

Separating variables and integrating on $[0, r]$ we have

$$
-\frac{\left(u(r)-\left(\frac{M}{C_{7}}\right)^{\frac{1}{q_{1}}}\right)^{1-\frac{q_{1}}{p-1}}-\left(d-\left(\frac{M}{C_{7}}\right)^{\frac{1}{q_{1}}}\right)^{1-\frac{q_{1}}{p-1}}}{\frac{p-1-q_{1}}{p-1}} \geq\left(\frac{C_{7}}{N}\right)^{\frac{1}{p-1}} \frac{r^{\frac{p}{p-1}}}{\frac{p}{p-1}},
$$

or equivalently, for all $r \in\left[0, r^{*}\right)$

$$
\left(u(r)-\left(\frac{M}{C_{7}}\right)^{\frac{1}{q_{1}}}\right)^{1-\frac{q_{1}}{p-1}}-\left(d-\left(\frac{M}{C_{7}}\right)^{\frac{1}{q_{1}}}\right)^{1-\frac{q_{1}}{p-1}} \geq C r^{\frac{p}{p-1}},
$$

where $C=C\left(p, q_{1}, N\right)=\frac{q_{1}+1-p}{p}\left(\frac{C_{7}}{N}\right)^{\frac{1}{p-1}}>0$. This and (1.3) yield

$$
\left(u(r)-\left(\frac{M}{C_{7}}\right)^{\frac{1}{q_{1}}}\right)^{-\frac{q_{1}+1-p}{p-1}} \geq C r^{\frac{p}{p-1}}, \quad \forall r \in\left[0, r^{*}\right) .
$$

Thus,

$$
u(r)-\left(\frac{M}{C_{7}}\right)^{\frac{1}{q_{1}}} \leq C_{1}\left(\frac{1}{r}\right)^{\frac{p}{q_{1}+1-p}}, \quad \forall r \in\left(0, r^{*}\right), \quad \text { where } C_{1}=\left(\frac{1}{C}\right)^{\frac{p-1}{q_{1}+1-p}} .
$$

Let $r \in\left[r_{1}, r^{*}\right)$. Replacing $\left(t_{1}, t_{2}\right)$ by $\left(r_{1}, r\right)$ in (5.11), from (5.7), (5.16), and (5.15),

$$
\begin{aligned}
P(r) & =r^{N}\left[\frac{p-1}{p}\left|u^{\prime}(r)\right|^{p}+G(u(r))\right]+\frac{N-p}{p} r^{N-1}\left|u^{\prime}(r)\right|^{p-2} u^{\prime}(r) u(r) \\
& \geq P\left(r_{1}\right)+\frac{N-p}{p} \int_{r_{1}}^{r} s^{N-1}\left[\tilde{C}_{1}(u(s))^{q_{1}+1}-M\right] \mathrm{d} s \geq P\left(r_{1}\right) \geq C d^{N\left[1-\frac{q_{1}+1}{p^{*}}\right] .}
\end{aligned}
$$

Since $u \geq 0$ and $u^{\prime} \leq 0$ on $\left[0, r^{*}\right)$,

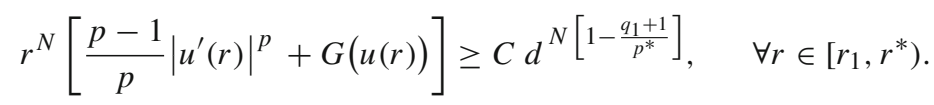

If the inequality

$$
G(u(\hat{r})) \geq \frac{p-1}{p}\left|u^{\prime}(\hat{r})\right|^{p},
$$

holds true for some $\hat{r} \in\left[r_{1}, r^{*}\right)$, then, from (5.9), and (5.19) we have

$$
\hat{r}^{N}\left[C_{6}|u(\hat{r})|^{q_{1}+1}+M\right] \geq \hat{r}^{N} G(u(\hat{r})) \geq \frac{C}{2} d^{N\left[1-\frac{q_{1}+1}{p^{*}}\right]} .
$$

This, and (5.17) imply

$$
C_{6} \hat{r}^{N}\left[C_{1}\left(\frac{1}{\hat{r}}\right)^{\frac{p}{q_{1}+1-p}}+\left(\frac{M}{C_{7}}\right)^{\frac{1}{q_{1}}}\right]^{q_{1}+1} \geq \frac{C}{2} d^{N\left[1-\frac{q_{1}+1}{p^{*}}\right]}-M \hat{r}^{N} \geq \frac{C}{4} d^{N\left[1-\frac{q_{1}+1}{p^{*}}\right]},
$$


and hence,

$$
C_{6}^{\frac{1}{q_{1}+1}} \hat{r}^{\frac{N}{q_{1}+1}}\left[C_{1}\left(\frac{1}{\hat{r}}\right)^{\frac{p}{q_{1}+1-p}}+\left(\frac{M}{C_{7}}\right)^{\frac{1}{q_{1}}}\right] \geq\left(\frac{C}{4}\right)^{\frac{1}{q_{1}+1}} d^{\frac{N}{q_{1}+1}\left[1-\frac{q_{1}+1}{p^{*}}\right]},
$$

and thus,

$$
\left(\frac{1}{\hat{r}}\right)^{\frac{p}{q_{1}+1-p}-\frac{N}{q_{1}+1}} \geq C d^{\frac{N}{q_{1}+1}\left[1-\frac{q_{1}+1}{p^{*}}\right]}
$$

Since $q_{1}$ is subcritical,

$$
\frac{p}{q_{1}+1-p}-\frac{N}{q_{1}+1}=\frac{1}{q_{1}+1} \frac{N p}{q_{1}+1-p}\left[1-\frac{q_{1}+1}{p^{*}}\right]>0 .
$$

Therefore, there exists a constant $C_{4}>0$ such that

$$
\hat{r} \leq C_{4}\left(\frac{1}{d}\right)^{\frac{q_{1}+1-p}{p}}, \quad \text { where } C_{4}=\left(\frac{1}{C}\right)^{\frac{p}{q_{1}+1-p}-\frac{N}{q_{1}+1}} .
$$

Let

$$
\tilde{r}:=C_{5}\left(\frac{1}{d}\right)^{\frac{q_{1}+1-p}{p}}, \quad \text { where } C_{5}:=\max \left\{C_{4}, 3 C_{2}\right\},
$$

and where $C_{2}$ is the constant in (3.9). If $r^{*} \leq \tilde{r}$, then we are done. So, we can assume that $r^{*}>\tilde{r}$. From (3.9), $\tilde{r}>3 C_{2}\left(\frac{1}{d}\right)^{\frac{q_{1}+1-p}{p}} \geq 2 r_{1}$. Thus, $r^{*}>2 r_{1}$ and (5.20) is false for all $r \in\left[\tilde{r}, r^{*}\right)$, then

$$
G(u(r)) \leq \frac{p-1}{p}\left|u^{\prime}(r)\right|^{p} \quad \forall r \in\left(\tilde{r}, r^{*}\right) .
$$

This and (5.19) show that there exists $K>0$ such that

$$
r^{N}\left|u^{\prime}(r)\right|^{p} \geq K d^{N\left[1-\frac{q_{1}+1}{p^{*}}\right]}, \quad \forall r \in\left[\tilde{r}, r^{*}\right) .
$$

Since $q_{1}$ is subcritical, see (3.24), $\frac{p}{q_{1}+1-p}>\frac{N}{q_{1}+1}>\frac{N-p}{p}$. Let $\tilde{M} \gg 1$ be a constant such that

$$
C_{1}\left(\frac{1}{\tilde{M}}\right)^{\frac{p}{q_{1}+1-p}}\left(\frac{1}{C_{5}}\right)^{\frac{p}{q_{1}+1-p}}-\frac{p K^{1 / p}}{(N-p) C_{5}^{(N-p) / p}}\left[1-\left(\frac{1}{2}\right)^{\frac{N-p}{p}}\right]\left(\frac{1}{\tilde{M}}\right)^{\frac{N-p}{p}}<0,
$$

where $C_{1}$ is as in (5.17), $C_{5}$ as in (5.26), and $K$ as in (5.28).

Suppose further that $r^{*}>2 \tilde{M} \tilde{r}$. From (5.28)

$$
-u^{\prime}(r) \geq K^{1 / p} d^{\frac{N}{p}\left[1-\frac{q_{1}+1}{p^{*}}\right]}\left(\frac{1}{r}\right)^{\frac{N}{p}}, \quad \forall r \in\left[\tilde{M} \tilde{r}, r^{*}\right) .
$$

Integrating on $[\tilde{M} \tilde{r}, r]$ for $r<r^{*}$, we have

$$
u(\tilde{M} \tilde{r})-u(r) \geq-\frac{p K^{1 / p}}{N-p} d^{\frac{N}{p}\left[1-\frac{q_{1}+1}{p^{*}}\right]}\left[\left(\frac{1}{r}\right)^{\frac{N}{p}-1}-\left(\frac{1}{\tilde{M} \tilde{r}}\right)^{\frac{N}{p}-1}\right], \quad \forall r \in\left[\tilde{M} \tilde{r}, r^{*}\right) .
$$


Therefore,

$$
u(r) \leq u(\tilde{M} \tilde{r})+C d^{\frac{N}{p}\left[1-\frac{q_{1}+1}{p^{*}}\right]}\left(\frac{1}{\tilde{M} \tilde{r}}\right)^{\frac{N}{p}-1}\left[\left(\frac{\tilde{M} \tilde{r}}{r}\right)^{\frac{N}{p}-1}-1\right], \quad \forall r \in\left[\tilde{M} \tilde{r}, r^{*}\right),
$$

where

$$
C:=\frac{p K^{1 / p}}{N-p}
$$

Moreover, from (5.26)

$$
d^{\frac{N}{p}\left[1-\frac{q_{1}+1}{p^{*}}\right]}\left(\frac{1}{\tilde{M} \tilde{r}}\right)^{\frac{N}{p}-1}=\left(\frac{1}{C_{5} \tilde{M}}\right)^{\frac{N-p}{p}} d^{\frac{N}{p}\left[1-\frac{q_{1}+1}{p^{*}}\right]+\frac{\left(q_{1}+1-p\right)}{p} \frac{(N-p)}{p}},
$$

and

$$
\frac{N}{p}\left[1-\frac{q_{1}+1}{p^{*}}\right]+\frac{\left(q_{1}+1-p\right)}{p} \frac{(N-p)}{p}=1 .
$$

Substituting inequality (5.17) in the first term of (5.30), (5.32) and (5.33) in the second term, by (5.29) and (5.31), we conclude that for all $r \in\left[2 \tilde{M} \tilde{r}, r^{*}\right)$

$$
\begin{aligned}
0 & \leq u(r) \leq\left(\frac{M}{C_{7}}\right)^{\frac{1}{q_{1}}}+C_{1}\left(\frac{1}{\tilde{M} \tilde{r}}\right)^{\frac{p}{q_{1}+1-p}}+C\left(\frac{1}{C_{5} \tilde{M}}\right)^{\frac{N-p}{p}}\left[\left(\frac{\tilde{M} \tilde{r}}{r}\right)^{\frac{N-p}{p}}-1\right] d \\
& \leq\left(\frac{M}{C_{7}}\right)^{\frac{1}{q_{1}}}+\left[C_{1}\left(\frac{1}{\tilde{M}}\right)^{\frac{p}{q_{1}+1-p}}\left(\frac{1}{C_{5}}\right)^{\frac{p}{q_{1}+1-p}}-\frac{C}{C_{5}^{\frac{N-p}{p}}}\left[1-\left(\frac{1}{2}\right)^{\frac{N-p}{p}}\right]\left(\frac{1}{\tilde{M}}\right)^{\frac{N-p}{p}}\right] d \\
& <0
\end{aligned}
$$

for $d$ big enough. This contradiction proves that for all $r<r^{*}, r \leq \max \left\{2 r_{1}, 2 \tilde{M} \tilde{r}\right\} \leq$ $C\left(\frac{1}{d}\right)^{\frac{q_{1}+1-p}{p}}$. Hence, $r^{*} \leq C\left(\frac{1}{d}\right)^{\frac{q_{1}+1-p}{p}}$. This implies $r^{*}<\infty$ and $r^{*}<\tau_{1}$. The proof of the claim is completed.

Let $r \in\left[r^{*}, \tau_{1}\right)$. From (2.10) and (5.1) we may write

$$
\begin{aligned}
r^{N-1}\left|u^{\prime}(r)\right|^{p-1} & =\int_{0}^{r_{1}} s^{N-1} g(u(s)) \mathrm{d} s+\int_{r_{1}}^{r} s^{N-1} g(u(s)) \mathrm{d} s \\
& \geq\left(C_{7}\left(\frac{d}{2}\right)^{q_{1}}-M\right) \frac{r_{1}^{N}}{N} \\
& \geq C d^{q_{1}} r_{1}^{N} .
\end{aligned}
$$

Hence, by using the estimate for $r_{1}$, we get

$$
-u^{\prime}(r) \geq C d^{\frac{p q_{1}-N\left(q_{1}+1-p\right)}{p(p-1)}} r^{(1-N) /(p-1)} .
$$

Letting $r=m r^{*}$ with $m>1$ and such that $m r^{*}<\tau_{1}$. Integration on $\left[r^{*}, r\right]$ yields

$$
\begin{aligned}
u\left(r^{*}\right) & \geq u\left(m r^{*}\right)+C d^{\frac{p q_{1}-N\left(q_{1}+1-p\right)}{p(p-1)}} \frac{p-1}{N-p}\left(\left(r^{*}\right)^{(p-N) /(p-1)}\left(1-m^{(p-N) /(p-1)}\right)\right) \\
& \geq C d\left(1-m^{(p-N) /(p-1)}\right) .
\end{aligned}
$$


From (5.16) and (5.35), it follows that, for $d>0$ large we have $m \leq 2$. This proves that $r<2 r^{*}$ which in turn proves that $\tau_{1}<2 r^{*} \leq C d^{\left(p-q_{1}-1\right) / p}$. Replacing this in (5.34) we have

$$
\left|u^{\prime}\left(\tau_{1}\right)\right|^{p-1} \geq C d^{q_{1}} \tau_{1} \geq C d^{\left(q_{1}+1\right)(p-1) / p},
$$

which implies $\left|u^{\prime}\left(\tau_{1}\right)\right| \geq C d^{\left(q_{1}+1\right) / p}$.

(ii) From (2.5) and (5.11) for $\left(t_{1}, t_{2}\right)=\left(0, \tau_{1}\right)$, we may write

$$
\begin{aligned}
P\left(\tau_{1}, d\right) & =\frac{p-1}{p} \tau_{1}^{N}\left|u^{\prime}\left(\tau_{1}\right)\right|^{p} \\
& =\frac{N-p}{p} \int_{0}^{\tau_{1}} s^{N-1}\left[p^{*} G(u(s))-g(u(s)) u(s)\right] \mathrm{d} s \\
& \leq \frac{N-p}{p} \int_{0}^{\tau_{1}} s^{N-1}\left[C_{1}(u(s))^{q_{1}+1}+M\right] \mathrm{d} s \\
& \leq C d^{q_{1}+1} \tau_{1}^{N}+\frac{M}{N} \tau_{1}^{N} \leq 2 C d^{q_{1}+1} \tau_{1}^{N},
\end{aligned}
$$

and therefore,

$$
\left|u^{\prime}\left(\tau_{1}\right)\right|^{p} \leq C d^{q_{1}+1} .
$$

The proof of the lemma is completed.

Remark 5.3 We show the changes in the proof of Lemma 3.3.

(i) Equations (3.42)-(3.45) and the arguments mentioned there, should be substituted by the following.

$$
-r^{N-1}\left|u^{\prime}(r)\right|^{p-2} u^{\prime}(r)=-\tau_{1}^{N-1}\left|u^{\prime}\left(\tau_{1}\right)\right|^{p-2} u^{\prime}\left(\tau_{1}\right)+\int_{\tau_{1}}^{r} s^{N-1} g(u(s)) \mathrm{d} s
$$

or, equivalently,

$$
r^{N-1}\left|u^{\prime}(r)\right|^{p-1}=\tau_{1}^{N-1}\left|u^{\prime}\left(\tau_{1}\right)\right|^{p-1}-\int_{\tau_{1}}^{r} s^{N-1}|g(u(s))| \mathrm{d} s .
$$

Assume that $\rho_{1}(d) \geq 3 \tau_{1}(d)$ and

$$
\int_{\tau_{1}}^{2 \tau_{1}} s^{N-1}|g(u(s))| \mathrm{d} s>\frac{1}{2} \tau_{1}^{N-1}\left|u^{\prime}\left(\tau_{1}\right)\right|^{p-1} .
$$

For any $\varepsilon>0$ small enough, there exists an $M_{\varepsilon}>0$ satisfying the following

$$
\begin{aligned}
\int_{2 \tau_{1}}^{3 \tau_{1}} s^{N-1}|g(u(s))| \mathrm{d} s & \geq \int_{2 \tau_{1}}^{3 \tau_{1}} s^{N-1}\left(\left(L_{2}-\varepsilon\right)|u(s)|^{q_{2}}-M_{\varepsilon}\right) \mathrm{d} s \\
& \geq\left(\left(L_{2}-\varepsilon\right)\left|u\left(2 \tau_{1}\right)\right|^{q_{2}}-M_{\varepsilon}\right) \frac{\left(3^{N}-2^{N}\right)}{N} \tau_{1}^{N} \\
& =\left(\left(L_{2}-\varepsilon\right)\left|u\left(2 \tau_{1}\right)\right|^{q_{2}}-M_{\varepsilon}\right) \frac{\left(3^{N}-2^{N}\right)}{\left(2^{N}-1\right)} \int_{\tau_{1}}^{2 \tau_{1}} s^{N-1} \mathrm{~d} s \\
& \geq \frac{\left(3^{N}-2^{N}\right)}{\left(2^{N}-1\right)} \int_{\tau_{1}}^{2 \tau_{1}} s^{N-1}\left(\left(L_{2}-\varepsilon\right)|u(s)|^{q_{2}}-M_{\varepsilon}\right) \mathrm{d} s
\end{aligned}
$$




$$
\begin{aligned}
& \geq \frac{\left(3^{N}-2^{N}\right)}{\left(2^{N}-1\right)} \int_{\tau_{1}}^{2 \tau_{1}} s^{N-1}\left(\frac{\left(L_{2}-\varepsilon\right)}{\left(L_{2}+\varepsilon\right)}|g(u(s))|-M^{\prime}\right) \mathrm{d} s \\
& \geq \frac{9}{8} \int_{\tau_{1}}^{2 \tau_{1}} s^{N-1}|g(u(s))| \mathrm{d} s-C M^{\prime} \tau_{1}^{N} \\
& \geq \int_{\tau_{1}}^{2 \tau_{1}} s^{N-1}|g(u(s))| \mathrm{d} s
\end{aligned}
$$

where we have used that $\frac{\left(3^{N}-2^{N}\right)}{\left(2^{N}-1\right)} \geq\left(\left(\frac{3}{2}\right)^{N}-1\right) \geq \frac{5}{4}$ for any $N \geq 2$, and $\varepsilon>0$ is fixed so that $\frac{5}{4} \frac{\left(L_{2}-\varepsilon\right)}{\left(L_{2}+\varepsilon\right)} \geq \frac{9}{8}$.

Furthermore, Eqs. (3.49)-(3.52) and the arguments involved, should be rewritten in the following way.

From (5.11),

$$
P(r, d)=P\left(\tau_{1}, d\right)+\frac{N-p}{p} \int_{\tau_{1}}^{r} s^{N-1}\left[p^{*} G(u(s))-g(u(s)) u(s)\right] \mathrm{d} s .
$$

From (2.5) and (5.8),

$$
\begin{aligned}
P(r, d) & \leq \frac{p-1}{p} \tau_{1}^{N}\left|u^{\prime}\left(\tau_{1}\right)\right|^{p}+\frac{N-p}{p} \int_{\tau_{1}}^{r} s^{N-1}\left[-C_{4}|u(s)|^{q_{2}+1}+M\right] \mathrm{d} s \\
& \leq \frac{p-1}{p} \tau_{1}^{N}\left|u^{\prime}\left(\tau_{1}\right)\right|^{p}+\frac{M}{p^{*}} r^{N}, \quad \forall r \in\left[\tau_{1}, \rho_{1}\right] .
\end{aligned}
$$

From this, (2.4), and (3.41),

$$
r^{N} G(u(r)) \leq P(r, d) \leq \frac{p-1}{p} \tau_{1}^{N}\left|u^{\prime}\left(\tau_{1}\right)\right|^{p}+\frac{M}{p^{*}} r^{N}, \quad \forall r \in\left[\tau_{1}, \rho_{1}\right] .
$$

And since (5.4), $C_{1}|u(r)|^{q_{2}+1} \leq C_{2}\left(\frac{\tau_{1}}{r}\right)^{N}\left|u^{\prime}\left(\tau_{1}\right)\right|^{p}+C_{3}$. Therefore,

$$
|u(r)|^{q_{2}+1} \leq C\left[1+\left|u^{\prime}\left(\tau_{1}\right)\right|^{p}\right], \quad \forall r \in\left[\tau_{1}, \rho_{1}\right],
$$

and using Lemma 3.2(ii),

$$
|u(r)|^{q_{2}+1} \leq C\left|u^{\prime}\left(\tau_{1}\right)\right|^{p}, \quad \forall r \in\left[\tau_{1}, \rho_{1}\right] .
$$

Integrating (3.47),

$$
|u(r)| \geq C \tau_{1}\left|u^{\prime}\left(\tau_{1}\right)\right|, \quad \forall r \in\left[\frac{3}{2} \tau_{1}, 2 \tau_{1}\right] .
$$

This, (5.45), and Lemma 3.2(i)-(ii) yield

$$
d^{q_{2}+1} \leq C\left(\tau_{1}\left|u^{\prime}\left(\tau_{1}\right)\right|\right)^{q_{2}+1} \leq C\left|u^{\prime}\left(\tau_{1}\right)\right|^{p} \leq C d^{q_{1}+1} .
$$

Now the facts $q_{2}>q_{1}$ and $d \gg 1$, provides the contradiction.

(ii) Equations (3.53)-(3.55) and the arguments involved contained in part (ii) of the proof, should be rewritten in the following way.

From (5.43),

$$
\rho_{1}^{N} G\left(u\left(\rho_{1}\right)\right)=P\left(\rho_{1}, d\right) \leq \frac{p-1}{p} \tau_{1}^{N}\left|u^{\prime}\left(\tau_{1}\right)\right|^{p}+\frac{M}{p^{*}} \rho_{1}^{N}
$$


Since (5.4), $C\left|u\left(\rho_{1}\right)\right|^{q_{2}+1}-M \leq\left(\frac{\tau_{1}}{\rho_{1}}\right)^{N}\left|u^{\prime}\left(\tau_{1}\right)\right|^{p}$. This, (3.37), and Lemma 3.2(ii) imply

$$
\left|u\left(\rho_{1}\right)\right| \leq C d^{\frac{q_{1}+1}{q_{2}+1}},
$$

for some constant $C>0$ independent of $d$.

Since (5.6)

$$
p^{*} G(u(s))-u(s) g(u(s)) \geq-\left(\left(L_{2}+\varepsilon\right)-\frac{p^{*}\left(L_{2}-\varepsilon\right)}{q_{2}+1}\right)|u(s)|^{q_{2}+1}-M_{\varepsilon}^{\prime \prime} .
$$

On the other hand, since (5.4), (5.42), and (5.8),

$$
\begin{aligned}
{\left[\frac{\left(L_{2}+\varepsilon\right)}{q_{2}+1}\left|u\left(\rho_{1}\right)\right|^{q_{2}+1}+M_{\varepsilon}^{\prime}\right] \rho_{1}^{N} } & \geq \rho_{1}^{N} G\left(u\left(\rho_{1}\right)\right)=P\left(\rho_{1}, d\right) \\
& \geq P\left(\tau_{1}, d\right)-\left[C_{3}\left|u\left(\rho_{1}\right)\right|^{q_{2}+1}+M\right] \frac{\rho_{1}^{N}-\tau_{1}^{N}}{N} .
\end{aligned}
$$

From (5.48),

$$
\left[C_{1}\left|u\left(\rho_{1}\right)\right|^{q_{2}+1}+C_{2}\right] \rho_{1}^{N} \geq \frac{p-1}{p} \tau_{1}^{N}\left|u^{\prime}\left(\tau_{1}\right)\right|^{p}+\left[C_{3}\left|u\left(\rho_{1}\right)\right|^{q_{2}+1}+M\right] \frac{\tau_{1}^{N}}{N} .
$$

Dividing by $\tau_{1}^{N},\left[C_{1}\left|u\left(\rho_{1}\right)\right|^{q_{2}+1}+C_{2}\right]\left(\rho_{1} / \tau_{1}\right)^{N} \geq \frac{p-1}{p}\left|u^{\prime}\left(\tau_{1}\right)\right|^{p}$.

From (3.16), (3.37), and Lemma 3.2(ii),

$$
\left|u\left(\rho_{1}\right)\right| \geq C d^{\frac{q_{1}+1}{q_{2}+1}}, \quad \text { for } d \gg 1,
$$

for some constant $C>0$ independent of $d$, ending this part of the proof.

(iii) Equations (3.56) and (3.57) should be rewritten in the following way. Let $r \in\left[r_{2}, \rho_{1}\right]$ [see (3.55)]. Integrating (2.9) on $\left[r, \rho_{1}\right]$,

$$
\begin{aligned}
-\left|u^{\prime}(r)\right|^{p-2} u^{\prime}(r) & =-\int_{r}^{\rho_{1}}\left(\frac{s}{r}\right)^{N-1} g(u(s)) \mathrm{d} s \\
& \geq \int_{r}^{\rho_{1}}\left(\frac{s}{r}\right)^{N-1}\left[C|u(s)|^{q_{2}}-M\right] \mathrm{d} s \\
& \geq\left[C \frac{\left|u\left(\rho_{1}\right)\right|^{q_{2}}}{2^{q_{2}}}-M\right]\left(\rho_{1}-r\right),
\end{aligned}
$$

and since Lemma 3.3(ii), $-\left|u^{\prime}(r)\right|^{p-2} u^{\prime}(r) \geq \frac{C}{2} \frac{\left|u\left(\rho_{1}\right)\right|^{q_{2}}}{2^{q_{2}}}\left(\rho_{1}-r\right)$, for $d \gg 1$, and therefore, $u^{\prime}(r) \leq 0$ and

$$
-u^{\prime}(r)=\left|u^{\prime}(r)\right| \geq C\left|u\left(\rho_{1}\right)\right|^{\frac{q_{2}}{p-1}}\left(\rho_{1}-r\right)^{\frac{1}{p-1}} .
$$

Remark 5.4 In a similar way, following the ideas of the previous remarks, it is possible to prove the extensions of Lemmas 3.4-3.6 to the more general nonlinearity $g$ satisfying hypothesis (1.4) of Remark 1.1(a). 


\section{References}

1. Castro, A., Kurepa, A.: Energy analysis of a nonlinear singular differential equations and applications. Rev. Colomb. Mat. 21, 155-166 (1987)

2. El Hachimi, A., De Thelin, F.: Infinitely many radially symmetric solutions for a quasilinear elliptic problem in a ball. J. Differ. Equ. 128, 78-102 (1996)

3. Castro, A., Kurepa, A.: Infinitely many radially symmetric solutions to a superlinear Dirichlet problem in a ball. Proc. Am. Math. Soc. 101(1), 57-64 (1987)

4. Castro, A., Kwon, J., Tan, C.M.: Infinitely many radial solutions for a sub-super critical Dirichlet boundary value problem in a ball. Electron. J. Differ. Equ. 2007(111), 1-10 (2007)

5. Kazdan, J., Warner, F.: Remarks on some quasilinear elliptic equations. Commun. Pure Appl. Math. 38, 557-569 (1975)

6. DiBenedetto, E.: $C^{1+\alpha}$ local regularity of weak solutions of degenerate elliptic equations. Nonlinear Anal. 7(8), 827-850 (1983)

7. Lieberman, G.M.: Boundary regularity for solutions of degenerate elliptic equations. Nonlinear Anal. 12(11), 1203-1219 (1988)

8. Pohozaev, S.I.: On the eigenfunctions of the equation $\Delta u+\lambda f(u)=0$. Dokl. Akad. Nauk SSSR 165, 36-39 (1965)

9. Bahri, A., Coron, J.M.: On a nonlinear elliptic equation involving the critical Sobolev exponent: the effect of the topology of the domain. Commun. Pure Appl. Math. 41(3), 253-294 (1988)

10. Pucci, P., Serrin, J.: The structure of the critical set in the mountain pass theorem. Trans. Am. Math. Soc. 299(1), 115-132 (1987)

11. Zou, H.H.: A priori estimates and existence for quasi-linear elliptic equations. Calc. Var. Partial Differ. Equ. 33(4), 417-437 (2008)

12. Damascelli, L., Pardo, R.: A priori estimates for some elliptic equations involving the $p$-Laplacian. Nonlinear Anal. 41(1), 475-496 (2018)

13. Cossio, J., Herrón, S.: Existence of radial solutions for an asymptotically linear $p$-Laplacian problem. J. Math. Anal. Appl. 345, 583-592 (2008)

14. Cossio, J., Herrón, S., Vélez, C.: Infinitely many radial solutions for a $p$-Laplacian problem $p$-superlinear at the origin. J. Math. Anal. Appl. 376, 741-749 (2011)

15. Fleckinger, J., Pardo, R., de Thélin, F.: Four-parameter bifurcation for a $p$-Laplacian system. Electron. J. Differ. Equ. 2001(6), 41-63 (2001)

16. Garcia Azorero, J., Peral Alonso, I.: Multiplicity of solutions for elliptic problems with critical exponent or with a nonsymmetric term. Trans. Am. Math. Soc. 323(2), 877-895 (1991)

17. Rabinowitz, P.H.: Minimax methods in critical point theory with applications to differential equations. In: CBMS Regional Conference Series in Mathematics, vol. 65 (1986)

18. García-Huidobro, M., Manásevich, R., Schmitt, K.: Positive radial solutions of quasilinear elliptic partial differential equations on a ball. Nonlinear Anal. 35(2), 175-190 (1999)

19. Castro, A., Pardo, R.: A priori bounds for positive solutions of subcritical elliptic equations. Rev. Mat. Complut. 28(3), 715-731 (2015)

20. Castro, A., Pardo, R.: A priori estimates for positive solutions to subcritical elliptic problems in a class of non-convex regions. Discrete Contin. Dyn. Syst. Ser. B 22(3), 783-790 (2017)

21. Mavinga, N., Pardo, R.: A priori bounds and existence of positive solutions for subcritical semilinear elliptic systems. J. Math. Anal. Appl. 449(2), 1172-1188 (2017)

22. Aguilar, J.A., Peral, I.: Positive radial solutions of quasilinear equations involving supercritical growth. NoDEA Nonlinear Differ. Equ. Appl. 5(3), 309-331 (1998)

23. Clapp, M., Tiwari, S.: Multiple solutions to a pure supercritical problem for the $p$-Laplacian. Calc. Var. Partial Differ. Equ. 55(1), 23 (2016). Art. 7

24. Clapp, M., Tiwari, S.: Existence and nonexistence of solutions to pure supercritical $p$-Laplacian problems. J. Fixed Point Theory Appl. 19(1), 375-385 (2017)

25. Il'yasov, Y., Runst, T.: Positive solutions of indefinite equations with $p$-Laplacian and supercritical nonlinearity. Complex Var. Elliptic Equ. 56(10-11), 945-954 (2011)

26. Iturriaga, L., Lorca, S., Massa, E.: Positive solutions for the $p$-Laplacian involving critical and supercritical nonlinearities with zeros. Ann. Inst. H. Poincaré C Anal. Non Linéaire 27(2), 763-771 (2010)

27. Zhao, L., Zhao, P.: The existence of three solutions for $p$-Laplacian problems with critical and supercritical growth. Rocky Mt. J. Math. 44(4), 1383-1397 (2014)

28. Zhong, Y.: Multiple solutions for the $p$-Laplacian problem with supercritical exponent. Bound. Value Probl. 2015(44), 15 (2015)

29. Bognár, G., Drábek, P.: The $p$-Laplacian equation with superlinear and supercritical growth, multiplicity of radial solutions. Nonlinear Anal. 60(4), 719-728 (2005) 
30. Dolbeault, J., Flores, I.: Geometry of phase space and solutions of semilinear elliptic equations in a ball. Trans. Am. Math. Soc. 359(9), 4073-4087 (2007)

31. Flores, I., Franca, M.: Phase plane analysis for radial solutions to supercritical quasilinear elliptic equations in a ball. Nonlinear Anal. 125, 128-149 (2015)

32. Herrón, S., Lopera, E.: Signed radial solutions for a weighted $p$-superlinear problem. Electron. J. Differ. Equ. 24, 1-13 (2014)

Publisher's Note Springer Nature remains neutral with regard to jurisdictional claims in published maps and institutional affiliations. 